\title{
On extensive form implementation of contracts in differential information economies ${ }^{\star}$
}

\author{
Dionysius Glycopantis ${ }^{1}$, Allan Muir ${ }^{2}$, and Nicholas C. Yannelis ${ }^{3}$ \\ 1 Department of Economics, City University, Northampton Square, London EC1V 0HB, UK \\ (e-mail: d.glycopantis@city.ac.uk) \\ 2 Department of Mathematics, City University, Northampton Square, London EC1V 0HB, UK \\ (e-mail: a.muir@city.ac.uk) \\ 3 Department of Economics, University of Illinois at Urbana-Champaign, IL 61820, USA \\ (e-mail: nyanneli@uiuc.edu)
}

Received: November 19, 2001; revised version: April 17, 2002

Summary. In the context of differential information economies, with and without free disposal, we consider the concepts of Radner equilibrium, rational expectations equilibrium, private core, weak fine core and weak fine value. We look into the possible implementation of these concepts as perfect Bayesian or sequential equilibria of noncooperative dynamic formulations. We construct relevant game trees which indicate the sequence of decisions and the information sets, and explain the rules for calculating ex ante expected payoffs. The possibility of implementing an allocation is related to whether or not it is incentive compatible. Implementation through an exogenous third party or an endogenous intermediary is also considered.

Keywords and Phrases: Differential information economy, Private core, Radner equilibrium, Rational expectations equilibrium, Weak fine core, Weak fine value, Free disposal, Coalitional Bayesian incentive compatibility, Game trees, Perfect Bayesian equilibrium, Sequential equilibrium, Contracts.

JEL Classification Numbers: 020, 226.

\section{Introduction}

An economy with differential information consists of a finite set of agents each of which is characterized by a random utility function, a random consumption set,

\footnotetext{
* This paper comes out of a visit by Nicholas Yannelis to City University, London, in December 2000. We are grateful to Dr A. Hadjiprocopis for his invaluable help with the implementation of Latex in a Unix environment. We also thank Leon Koutsougeras and a referee for several, helpful comments. Correspondence to: N. C. Yannelis
} 
random initial endowments, a private information set defined on the states of nature, and a prior probability distribution on these states. For such an economy there are a number of cooperative and non-cooperative equilibrium concepts.

We have the noncooperative concepts of the generalized Walrasian equilibrium ideas of Radner equilibrium and rational expectations equilibrium (REE) defined in Radner (1968), Allen (1981) and Einy, Moreno, and Shitovitz (2000, 2001). ${ }^{1}$ We also have the cooperative concepts of the private core (Yannelis, 1991), of the weak fine core, defined in Yannelis (1991) and Koutsougeras and Yannelis (1993), and that of the weak fine value (Krasa and Yannelis, 1994). The last two concepts allow the agents to pool their information. ${ }^{2}$

In a comparison of the equilibrium concepts we note that contrary to the private core any rational expectations Walrasian equilibium notion will always give zero quantities to an agent whose initial endowments are zero in each state. This is so irrespective of whether his private information is the full partition or the trivial partition of the states of nature. Hence the Radner as well as the REE do not register the informational superiority of an agent.

In Glycopantis, Muir, and Yannelis (2001) we provided a noncooperative interpretation of the private core for a three persons economy without free disposal. We constructed game trees which indicate the sequence of decisions and the information of the agents, and explained the rules for calculating ex ante, expected payoffs, through the reallocation of initial endowments. We showed that the private core can be given a dynamic interpretation as a perfect Bayesian equilibrium (PBE) of a noncooperative extensive form game.

The term implementation is used in the sense of realization of an allocation and not in the formal sense of implementation theory or mechanism design. Implementation or support of an allocation is sought through the PBE concept, described in Tirole (1988), which is a variant of the Kreps-Wilson (1982) idea of sequential equilibrium.

A PBE consists of a set of players' optimal behavioral strategies, and consistent with these, a set of beliefs which attach a probability distribution to the nodes of each information set. Consistency requires that the decision from an information set is optimal given the particular player's beliefs about the nodes of this set and the strategies from all other sets, and that beliefs are formed from updating, using the available information. If the optimal play of the game enters an information set then updating of beliefs must be Bayesian. Otherwise appropriate beliefs are assigned arbitrarily to the nodes of the set. This equilibrium concept is further looked at in Appendix I.

Our main observation in Glycopantis, Muir, and Yannelis (2001) was that Bayesian incentive compatible concepts, like the private core, can be implemented as a PBE of a noncooperative, extensive form game. Moreover we provided a counter example which demonstrates that core concepts which are not necessarily Bayesian incentive compatible, as for example the weak fine core, cannot be supported, under reasonable rules, in a dynamic framework. In the present paper we

\footnotetext{
${ }^{1}$ Kurz (1994) has provided the alternative idea of rational belief equilibria.

2 See also Allen and Yannelis (2001) for additional references.
} 
examine further the issue of extensive form implementation and obtain additional results.

Firstly, we consider cooperative and noncooperative solution concepts with and without free disposal. To our surprise, as it was not intuitively obvious, we found that solution concepts which are Bayesian incentive compatible without free disposal, do not retain this property under free disposal. In particular, not only free disposal destroys incentive compatibility but a problem also appears in verifying that an agent has actually destroyed part of his initial endowment.

Secondly, we provide examples which demonstrate that with free disposal cooperative and noocooperative solution concepts are not implementable as a PBE. However implementation becomes possible by introducing a third party, such as a court which has perfect knowledge in order to be able to penalize the lying agents.

Thirdly, for the purpose of implementation of the (non-free) disposal private core, we follow an alternative approach. We consider the (non-free) disposal private core example of the one-good, three-agent economy discussed in Glycopantis, Muir, and Yannelis (2001). The introduction of a third party results in the implementation of the private core allocation as a PBE. We show here that it can also be implemented as a sequential equilibrium (Kreps and Wilson, 1982).

Finally we provide a full characterization of our Bayesian incentive compatibility concept in the case of one good per state.

The analysis suggests that if an allocation is not incentive compatible, i.e. the agents do not find that it is in accordance with their interests, then there is a difficulty in implementing it in a dynamic framework. On the other hand incentive compatible allocations are implementable through contracts with reasonable conditions. We note that the implementation analysis is independent of the equilibrium notion. It applies to contracts in general which can be analysed by a similar tree structure.

Parts of the investigation fall into the area of the Nash programme the purpose of which has been, as explained in Glycopantis, Muir, and Yannelis (2001), to provide support and justification of cooperative solutions through noncooperative formulations. On the other hand we extend here the investigation into more general areas by discussing explicitly the possible implementation of noncooperative concepts such as Radner equilibrium and REE. It appears that in general the issue is the relation between dynamic and static considerations, not necessarily between cooperative and noncooperative formulations.

The paper is organized as follows. Section 2 defines a differential information exchange economy. Section 3 contains the equilibrium concepts discussed in this paper. Section 4 describes ideas of incentive compatibility. Section 5 discusses the non-implementation of free disposal private core allocations and Section 6 the implementation of private core and Radner equilibria through the courts. Section 7 discusses the implementation of non-free disposal private core allocations through an endogenous intermediary. Section 8 offers concluding remarks. Appendix I contains further remarks on PBE. 


\section{Differential information economy}

We define the notion of a finite-agent economy with differential information, confining ourselves to the case where the set of states of nature, $\Omega$, is finite and there is a finite number of goods, $l$, per state. $\mathcal{F}$ is a $\sigma$-algebra on $\Omega, I$ is a set of $n$ players and $\mathbb{R}_{+}^{l}$ will denote the positive orthant of $\mathbb{R}^{l}$.

A differential information exchange economy $\mathcal{E}$ is a set $\left\{\left((\Omega, \mathcal{F}), X_{i}, \mathcal{F}_{i}, u_{i}\right.\right.$, $\left.\left.e_{i}, q_{i}\right): i=1, \ldots, n\right\}$ where

1. $X_{i}: \Omega \rightarrow 2^{\mathbb{R}_{+}^{l}}$ is the set-valued function giving the random consumption set of Agent (Player) i, who is denoted also by Pi;

2. $\mathcal{F}_{i}$ is a partition of $\Omega$, denoting the private information ${ }^{3}$ of $\mathrm{Pi}$;

3. $u_{i}: \Omega \times \mathbb{R}_{+}^{l} \rightarrow \mathbb{R}$ is the random utility function of $\mathrm{Pi}$;

4. $e_{i}: \Omega \rightarrow \mathbb{R}_{+}^{l}$ is the random initial endowment of $\mathrm{Pi}$, assumed to be constant on elements of $\mathcal{F}_{i}$, with $e_{i}(\omega) \in X_{i}(\omega)$ for all $\omega \in \Omega$;

5. $q_{i}$ is an $\mathcal{F}$-measurable probability function on $\Omega$ giving the prior of Pi. It is assumed that on all elements of $\mathcal{F}_{i}$ the aggregate $q_{i}$ is positive. If a common prior is assumed it will be denoted by $\mu$.

We will refer to a function with domain $\Omega$, constant on elements of $\mathcal{F}_{i}$, as $\mathcal{F}_{i}$-measurable, although, strictly speaking, measurability is with respect to the $\sigma$ algebra generated by the partition. We can think of such a function as delivering information to Pi which does not permit discrimination between the states of nature belonging to any element of $\mathcal{F}_{i}$.

In the first period agents make contracts in the ex ante stage. In the interim stage, i.e., after they have received a signal ${ }^{4}$ as to what is the event containing the realized state of nature, one considers the incentive compatibility of the contract.

For any $x_{i}: \Omega \rightarrow \mathbb{R}_{+}^{l}$, the ex ante expected utility of $\mathrm{Pi}$ is given by

$$
v_{i}\left(x_{i}\right)=\sum_{\omega \in \Omega} u_{i}\left(\omega, x_{i}(\omega)\right) q_{i}(\omega) .
$$

Denote by $E_{i}(\omega)$ the element in the partition $\mathcal{F}_{i}$ which contains the realized state of nature, $\omega \in \Omega$. It is assumed that $q_{i}\left(E_{i}(\omega)\right)>0$ for all $\omega \in \Omega$. The interim expected utility function of $\mathrm{Pi}$ is given by

$$
v_{i}\left(\omega, x_{i}\right)=\sum_{\omega^{\prime} \in \Omega} u_{i}\left(\omega^{\prime}, x_{i}\left(\omega^{\prime}\right)\right) q_{i}\left(\omega^{\prime} \mid E_{i}(\omega)\right),
$$

where

$$
q_{i}\left(\omega^{\prime} \mid E_{i}(\omega)\right)=\left\{\begin{array}{lll}
0 & \text { for } \quad \omega^{\prime} \notin E_{i}(\omega) \\
\frac{q_{i}\left(\omega^{\prime}\right)}{q_{i}\left(E_{i}(\omega)\right)} & \text { for } \quad \omega^{\prime} \in E_{i}(\omega) .
\end{array}\right.
$$

${ }^{3}$ Following Aumann (1987) we assume that the players' information partitions are common knowledge. Sometimes $\mathcal{F}_{i}$ will denote the $\sigma$-algebra generated by the partition, in which case $\mathcal{F}_{i} \subseteq \mathcal{F}$, as it will be clear from the context.

${ }^{4}$ A signal to $\mathrm{Pi}$ is an $\mathcal{F}_{i}$-measurable function from $\Omega$ to the set of the possible distinct observations specific to the player; that is, it induces the partition $\mathcal{F}_{i}$, and so gives the finest discrimination of states of nature directly available Pi. 


\section{Private core, weak fine core, Radner equilibrium, REE and weak fine value}

We define here the various equilibrium concepts in this paper, distinguishing between the free disposal and the non-free disposal case. A comparison is also made between these concepts. All definitions are in the context of the exchange economy $\mathcal{E}$ in Section 2.

We begin with some notation. Denote by $L_{1}\left(q_{i}, \mathbb{R}^{l}\right)$ the space of all equivalence classes, with respect to $q_{i}$, of $\mathcal{F}$-measurable functions $f_{i}: \Omega \rightarrow \mathbb{R}^{l}$.

$L_{X_{i}}$ is the set of all $\mathcal{F}_{i}$-measurable selections from the random consumption set of Agent i, i.e.,

$$
\begin{gathered}
L_{X_{i}}=\left\{x_{i} \in L_{1}\left(q_{i}, \mathbb{R}^{l}\right): x_{i}: \Omega \rightarrow \mathbb{R}^{l} \text { is } \mathcal{F}_{i}\right. \text {-measurable } \\
\text { and } \left.x_{i}(\omega) \in X_{i}(\omega) q_{i} \text {-a.e. }\right\}
\end{gathered}
$$

and let $L_{X}=\prod_{i=1}^{n} L_{X_{i}}$.

Also let

$$
\bar{L}_{X_{i}}=\left\{x_{i} \in L_{1}\left(q_{i}, \mathbb{R}^{l}\right): x_{i}(\omega) \in X_{i}(\omega) q_{i} \text {-a.e. }\right\}
$$

and let $\bar{L}_{X}=\prod_{i=1}^{n} \bar{L}_{X_{i}}$.

An element $x=\left(x_{1}, \ldots, x_{n}\right) \in \bar{L}_{X}$ will be called an allocation. For any subset of players $S$, an element $\left(y_{i}\right)_{i \in S} \in \prod_{i \in S} \bar{L}_{X_{i}}$ will also be called an allocation, although strictly speaking it is an allocation to $S$.

We note that the above notation is employed also for purposes of comparisons with the analysis in Glycopantis, Muir, and Yannelis (2001). In case there is only one good, i.e. $l=1$, we shall use the notation $L_{X_{i}}^{1}, \bar{L}_{X_{i}}^{1}$ etc. When a common prior is also assumed $L_{1}\left(q_{i}, \mathbb{R}^{l}\right)$ will be replaced by $L_{1}\left(\mu, \mathbb{R}^{l}\right)$.

First we define the notion of the (ex ante) private core ${ }^{5}$ (Yannelis, 1991).

Definition 3.1. An allocation $x \in L_{X}$ is said to be a private core allocation if

(i) $\sum_{i=1}^{n} x_{i}=\sum_{i=1}^{n} e_{i}$ and

(ii) there do not exist coalition $S$ and allocation $\left(y_{i}\right)_{i \in S} \in \prod_{i \in S} L_{X_{i}}$ such that $\sum_{i \in S} y_{i}=\sum_{i \in S} e_{i}$ and $v_{i}\left(y_{i}\right)>v_{i}\left(x_{i}\right)$ for all $i \in S$.

Notice that the definition above does not allow for free disposal. If the feasibility condition (i) is replaced by (i) ${ }^{\prime} \sum_{i=1}^{n} x_{i} \leq \sum_{i=1}^{n} e_{i}$ then free disposal is allowed.

Example 3.1. Consider the following three agents economy, $I=\{1,2,3\}$ with one commodity, i.e. $X_{i}=\mathbb{R}_{+}$for each i, and three states of nature $\Omega=\{a, b, c\}$.

We assume that the initial endowments and information partitions of the agents are given by

5 The private core can also be defined as an interim concept. See Yannelis (1991) and Glycopantis, Muir, and Yannelis (2001). 


$$
\begin{array}{ll}
e_{1}=(5,5,0), & \mathcal{F}_{1}=\{\{a, b\},\{c\}\} ; \\
e_{2}=(5,0,5), & \mathcal{F}_{2}=\{\{a, c\},\{b\}\} ; \\
e_{3}=(0,0,0), & \mathcal{F}_{3}=\{\{a\},\{b\},\{c\}\} .
\end{array}
$$

It is also assumed that $u_{i}\left(\omega, x_{i}(\omega)\right)=x_{i}^{\frac{1}{2}}$, which is a typical strictly concave and monotone function in $x_{i}$, and that every player expects that each state of nature occurs with the same probability, i.e. $\mu(\{\omega\})=\frac{1}{3}$, for $\omega \in \Omega$. For convenience, in the discussion below expected utilities are multiplied by 3 .

It was shown in Appendix II of Glycopantis, Muir, and Yannelis (2001) that, without free disposal, a private core allocation of this economy is $x_{1}=(4,4,1)$, $x_{2}=(4,1,4)$ and $x_{3}=(2,0,0)$. It is important to observe that in spite of the fact that Agent 3 has zero initial endowments, his superior information allows him to make a Pareto improvement for the economy as a whole and he was rewarded for doing so. In other words, Agent 3 traded his superior information for actual consumption in state $a$. In return Agent 3 provided insurance to Agent 1 in state $c$ and to Agent 2 in state $b$. Notice that if the private information set of Agent 3 is the trivial partition, i.e., $\mathcal{F}_{3}^{\prime}=\{a, b, c\}$, then no-trade takes place and clearly in this case he gets zero utility. Thus the private core is sensitive to information asymmetries.

Next we define another core concept, the weak fine core (Yannelis, 1991; Koutsougeras and Yannelis, 1993). This is a refinement of the fine core concept of Wilson (1978). Recall that the fine core notion of Wilson as well as the fine core in Koutsougeras and Yannelis may be empty in well behaved economies. It is exactly for this reason that we are working with a different concept.

Definition 3.2. An allocation $x=\left(x_{1}, \ldots, x_{n}\right) \in \bar{L}_{X}$ is said to be a weak fine core allocation if

(i) each $x_{i}(\cdot)$ is $\bigvee^{n} \mathcal{F}_{i}$-measurable ${ }^{6}$

(ii) $\sum_{i=1}^{n} x_{i}=\sum_{i=1}^{n} e_{i}$ and

(iii) there do not exist coalition $S$ and allocation $\left(y_{i}\right)_{i \in S} \in \prod_{i \in S} \bar{L}_{X_{i}}$ such that $y_{i}(\cdot)-e_{i}(\cdot)$ is $\bigvee_{i \in S} \mathcal{F}_{i}$-measurable for all $i \in S, \sum_{i \in S} y_{i}=\sum_{i \in S} e_{i}$ and $v_{i}\left(y_{i}\right)>$ $v_{i}\left(x_{i}\right)$ for all $i \in S$.

Existence of private core and weak fine core allocations is discussed in Glycopantis, Muir, and Yannelis (2001). The weak fine core is also an ex ante concept. As with the private core the feasibility condition can be relaxed to (ii) ${ }^{\prime} \sum_{i=1}^{n} x_{i} \leq$ $\sum_{i=1}^{n} e_{i}$. Notice however that now coalitions of agents are allowed to pool their own information and all alocations will exhaust the resource. The example below illustrates this concept.

Example 3.2. Consider the Example 3.1 without Agent 3. Then if Agents 1 and 2 pool their own information a possible allocation is $x_{1}=x_{2}=(5,2.5,2.5)$. Notice

$6 \bigvee_{i=1}^{n} \mathcal{F}_{i}$ denotes the smallest $\sigma$-algebra containing each $\mathcal{F}_{i}$. 
that this allocation is $\bigvee_{i=1}^{2} \mathcal{F}_{i}$-measurable and cannot be dominated by any coalition of agents using their pooled information. Hence it is a weak fine core allocation. ${ }^{7}$

Next we shall define a Walrasian equilibrium notion in the sense of Radner. In order to do so, we need the following. A price system is an $\mathcal{F}$-measurable, nonzero function $p: \Omega \rightarrow \mathbb{R}_{+}^{l}$ and the budget set of Agent $\mathrm{i}$ is given by

$$
\begin{aligned}
B_{i}(p)=\left\{x_{i}: x_{i}: \Omega \rightarrow\right. & \mathbb{R}^{l} \text { is } \mathcal{F}_{i} \text {-measurable } x_{i}(\omega) \in X_{i}(\omega) \\
& \text { and } \left.\sum_{\omega \in \Omega} p(\omega) x_{i}(\omega) \leq \sum_{\omega \in \Omega} p(\omega) e_{i}(\omega)\right\} .
\end{aligned}
$$

Notice that the budget constraint is across states of nature.

Definition 3.3. A pair $(p, x)$, where $p$ is a price system and $x=\left(x_{1}, \ldots, x_{n}\right) \in$ $L_{X}$ is an allocation, is a Radner equilibrium if

(i) for all $\mathrm{i}$ the consumption function maximizes $v_{i}$ on $B_{i}$

(ii) $\sum_{i=1}^{n} x_{i} \leq \sum_{i=1}^{n} e_{i}$ (free disposal), and

(iii) $\sum_{\omega \in \Omega} p(\omega) \sum_{i=1}^{n} x_{i}(\omega)=\sum_{\omega \in \Omega} p(\omega) \sum_{i=1}^{n} e_{i}(\omega)$.

Radner equilibrium is an ex ante concept. We assume free disposal, for otherwise it is well known that a Radner equilibrium with non-negative prices might not exist. This can be seen through straightforward calculations in Example 3.1.

Next we turn our attention to the notion of REE. We shall need the following. Let $\sigma(p)$ be the smallest sub- $\sigma$-algebra of $\mathcal{F}$ for which $p: \Omega \rightarrow \mathbb{R}_{+}^{l}$ is measurable and let $\mathcal{G}_{i}=\sigma(p) \vee \mathcal{F}_{i}$ denote the smallest $\sigma$-algebra containing both $\sigma(p)$ and $\mathcal{F}_{i}$. We shall also condition the expected utility of the agents on $\mathcal{G}_{i}$ which produces a random variable.

Definition 3.4. A pair $(p, x)$, where $p$ is a price system and $x=\left(x_{1}, \ldots, x_{n}\right) \in$ $\bar{L}_{X}$ is an allocation, is a rational expectations equilibrium (REE) if

(i) for all $i$ the consumption function $x_{i}(\omega)$ is $\mathcal{G}_{i}$-measurable.

(ii) for all $\mathrm{i}$ and for all $\omega$ the consumption function maximizes

$$
v_{i}\left(x_{i} \mid \mathcal{G}_{i}\right)(\omega)=\sum_{\omega^{\prime} \in E_{i}^{\mathcal{G}_{i}}(\omega)} u_{i}\left(\omega^{\prime}, x_{i}\left(\omega^{\prime}\right)\right) \frac{q_{i}\left(\omega^{\prime}\right)}{q_{i}\left(E_{i}^{\mathcal{G}_{i}}(\omega)\right)},
$$

(where $E_{i}^{\mathcal{G}_{i}}(\omega)$ is the event in $\mathcal{G}_{i}$ which contains $\omega$ and $q_{i}\left(E_{i}^{\mathcal{G}_{i}}(\omega)\right)>0$ ) subject to

$$
p(\omega) x_{i}(\omega) \leq p(\omega) e_{i}(\omega)
$$

i.e. the budget set at state $\omega$, and

(iii) $\sum_{i=1}^{n} x_{i}(\omega)=\sum_{i=1}^{n} e_{i}(\omega)$ for all $\omega$.

7 See Koutsougeras and Yannelis (1993). 
This is an interim concept because we condition expectations on information received from prices as well. In the definition, free disposal can easily be introduced. The idea of conditioning on the $\sigma$-algebra, $v_{i}\left(x_{i} \mid \mathcal{G}_{i}\right)(\omega)$, is rather well known.

REE can be classified as (i) fully revealing if the price function reveals to each agent all states of nature, (ii) partially revealing if the price function reveals some but not all states of nature and (iii) non-revealing if it does not disclose any particular state of nature.

Finally we define the concept of weak fine value allocation (see Krasa and Yannelis, 1994). As in the definition of the standard value allocation concept, we must first define a transferable utility (TU) game in which each agent's utility is weighted by a factor $\lambda_{i}(i=1, \ldots, n)$, which allows interpersonal comparisons. In the value allocation itself no side payments are necessary. ${ }^{8}$ A game with side payments is then defined as follows.

Definition 3.5. A game with side payments $\Gamma=(I, V)$ consist of a finite set of agents $I=\{1, \ldots, n\}$ and a superadditive, real valued function $V$ defined on $2^{I}$ such that $V(\emptyset)=0$. Each $S \subset I$ is called a coalition and $V(S)$ is the 'worth' of the coalition $S$.

The Shapley value of the game $\Gamma$ (Shapley, 1953) is a rule that assigns to each Agent $i$ a 'payoff', $S h_{i}$, given by the formula ${ }^{9}$

$$
S h_{i}(V)=\sum_{\substack{S \subseteq I \\ S \supseteq\{i\}}} \frac{(|S|-1) !(|I|-|S|) !}{|I| !}[V(S)-V(S \backslash\{i\})] .
$$

The Shapley value has the property that $\sum_{i \in I} S h_{i}(V)=V(I)$, i.e. it is Pareto efficient.

We now define for each economy with differential information, $\mathcal{E}$, and a common prior, and for each set of weights, $\lambda_{i}: i=1, \ldots, n$, the associated game with side payments $\left(I, V_{\lambda}\right)$ (we also refer to this as a 'transferable utility' (TU) game) as follows:

For every coalition $S \subset I$, let

$$
V_{\lambda}(S)=\max _{x} \sum_{i \in S} \lambda_{i} \sum_{\omega \in \Omega} u_{i}\left(\omega, x_{i}(\omega)\right) \mu(\omega)
$$

subject to

(i) $\sum_{i \in S} x_{i}(\omega)=\sum_{i \in S} e_{i}(\omega), \mu-$ a.e.,

(ii) $x_{i}-e_{i}$ is $\bigvee_{i \in S} \mathcal{F}_{i}$-measurable.

We are now ready to define the weak fine value allocation.

\footnotetext{
8 See Emmons and Scafuri (1985, p. 60) for further discussion.

9 The Shapley value measure is the sum of the expected marginal contributions an agent can make to all the coalitions of which he/she is a member (see Shapley, 1953).
} 
Definition 3.6. An allocation $x=\left(x_{1}, \ldots, x_{n}\right) \in \bar{L}_{X}$ is said to be a weak fine value allocation of the differential information economy, $\mathcal{E}$, if the following conditions hold

(i) Each net trade $x_{i}-e_{i}$ is $\bigvee_{i=1}^{n} \mathcal{F}_{i}$-measurable,

(ii) $\sum_{i=1}^{n} x_{i}=\sum_{i=1}^{n} e_{i}$ and

(iii) There exist $\lambda_{i} \geq 0$, for every $i=1, \ldots, n$, which are not all equal to zero, with $\sum_{\omega \in \Omega} \lambda_{i} u_{i}\left(\omega, x_{i}(\omega)\right) \mu(\omega)=S h_{i}\left(V_{\lambda}\right)$ for all $i$, where $S h_{i}\left(V_{\lambda}\right)$ is the Shapley value of Agent $i$ derived from the game $\left(I, V_{\lambda}\right)$, defined in (5) above.

Condition (i) requires the pooled information measurability of net trades, i.e. net trades are measurable with respect to the "join". Condition (ii) is the market clearing condition and (iii) says that the expected utility of each agent multiplied by his/her weight, $\lambda_{i}$, must be equal to his/her Shapley value derived from the TU game $\left(I, V_{\lambda}\right)$.

An immediate consequence of Definition 3.6 is that

$$
S h_{i}\left(V_{\lambda}\right) \geq \lambda_{i} \sum_{\omega \in \Omega} u_{i}\left(\omega, e_{i}(\omega)\right) \mu(\omega)
$$

for every $i$, i.e. the value allocation is individually rational. This follows immediately from the fact that the game $\left(V_{\lambda}, I\right)$ is superadditive for all weights $\lambda$. Similarly, efficiency of the Shapley value for games with side payments immediately implies that the value allocation is weak-fine Pareto efficient.

On the basis of the definitions and the analysis of Example 3.1 of an exhange economy with 3 agents and of Example 3.2 with 2 agents we make comparisons between the various equilibrium notions. The calculations of all, cooperative and noncooperative, equilibrium allocations are straightforward.

Contrary to the private core any rational expectation Walrasian equilibium notion, such as Radner equilibrium or REE, will always give zero to an agent who has no initial endowments. For example, in the 3-agent economy of Example 3.1, Agent 3 receives no consumption since his budget set is zero in each state. This is so irrespective of whether his private information is the full information partition $\mathcal{F}_{3}=\{\{a\},\{b\},\{c\}\}$ or the trivial partition $\mathcal{F}_{3}^{\prime}=\{a, b, c\}$. Hence the Walrasian, competitive equilibrium ideas do not take into account the informational superiority of an agent.

The set of Radner equilibrium allocations, with and without free disposal, are a subset of the corresponding private core allocations. Of course it is possible that a Radner equilibrium allocation might not exist. In the two-agent economy of Example 3.2, assuming non-free disposal the unique private core is the initial endowments allocation while no Radner equilibrium exists. On the other hand, assuming free disposal, for the same example, the REE coincides with the initial endowments allocation which does not belong to the private core. It follows that the REE allocations need not be in the private core.

We also have that a REE need not be a Radner equilibrium. In Example 3.2, without free disposal no Radner equilibrium with non-negative prices exists but REE does. It is unique and it implies no-trade. 
As for the comparison between private and weak fine core allocations the two sets could intersect but there is no definite relation. Indeed the measurability requirement of the private core allocations separates the two concepts. In Example 3.2 the allocation $(5,2.5,2.5)$ to Agent 1 and $(5,2.5,2.5)$ to Agent 2 , as well as $(6$, $3,3)$ and $(4,2,2)$ belong to the weak fine core but not to the private core. There are many weak fine core allocations which do not satisfy the measurability condition.

For $n=2$ one can easily verify that the weak fine value belongs to the weak fine core. However it is known (see, for example, Scafuri and Yannelis, 1984) that for $n \geq 3$ a value allocation may not be a core allocation, and therefore may not be a Radner equilibrium.

Also, in Example 3.1 a private core allocation is not necessarily in the weak fine core. Indeed the division $(4,4,1),(4,1,4)$ and $(2,0,0)$, to Agents 1,2 and 3 respectively, is a private core but not a weak fine core allocation. The first two agents can get together, pool their information and do better. They can realize the weak fine core allocation, $(5,2.5,2.5),(5,2.5,2.5)$ and $(0,0,0)$ which does not belong to the private core.

Finally notice that even with free disposal no allocation which does not distribute the total resource could be in the weak fine core. The three agents can get together, distribute the surplus and increase their utility.

In the next section we shall discuss whether core and Walrasian type allocations have certain desirable properties from the point of view of incentive compatibility. Following this, we shall turn our attention in later sections to the implementation of such allocations.

\section{Incentive compatibility}

The basic idea is that an allocation is incentive compatible if no coalition can misreport the realized state of nature to the complementary set of agents and become better off.

Let us suppose we have a coalition $S$, with members denoted by $i$, and the complementary set $I \backslash S$ with members $j$. Let the realized state of nature be $\omega^{*}$. A member $i \in S$ sees $E_{i}\left(\omega^{*}\right)$. Obviously not all $E_{i}\left(\omega^{*}\right)$ need be the same, however all Agents $i$ know that the actual state of nature could be $\omega^{*}$.

Consider now a state of nature $\omega^{\prime}$ with the following property. For all $j \in I \backslash S$ we have $\omega^{\prime} \in E_{j}\left(\omega^{*}\right)$ and for at least one $i \in S$ we have $\omega^{\prime} \notin E_{i}\left(\omega^{*}\right)$ (otherwise $\omega^{\prime}$ would be indistinguishable from $\omega^{*}$ for all players and, by redefining utilities appropriately, could be considered as the same element of $\Omega$ ). Now the coalition $S$ decides that each member $i$ will announce that she has seen her own set $E_{i}\left(\omega^{\prime}\right)$ which, of course, definitely contains a lie. On the other hand we have that $\omega^{\prime} \in$ $\bigcap E_{j}\left(\omega^{*}\right)$, (we also denote $j \in I \backslash S$ by $j \notin S$ ). $j \notin S$

Now the idea is that if all members of $I \backslash S$ believe the statements of the members of $S$ then each $i \in S$ expects to gain. For coalitional Bayesian incentive compatibility (CBIC) of an allocation we require that this is not possible. This is the incentive compatibility condition used in Glycopantis, Muir, and Yannelis (2001) where we gave a formal definition. 
We showed there that in the three-agent economy without free disposal the private core allocation $x_{1}=(4,4,1), x_{2}=(4,1,4)$ and $x_{3}=(2,0,0)$ is incentive compatible. This follows from the fact that Agent 3 who would potentially cheat in state $a$ has no incentive to do so. It has been shown in Koutsougeras and Yannelis (1993) that if the utility functions are monotone and continuous then private core allocations are always CBIC.

On the other hand the weak fine core allocations are not always incentive compatible, as the proposed redistribution $x_{1}=x_{2}=(5,2.5,2.5)$ in the two-agent economy shows. Indeed, if Agent 1 observes $\{a, b\}$, he has an incentive to report $c$ and Agent 2 has an incentive to report $b$ when he observes $\{a, c\}$.

CBIC coincides in the case of a two-agent economy with Individually Bayesian Incentive Compatibility (IBIC) which corresponds to the case in which $S$ is a singleton.

The concept of Transfer Coalitionally Bayesian Incentive Compatible (TCBIC) allocations, used in this paper, ${ }^{10}$ allows for transfers between the members of a coalition, and is therefore a strengthening of the concept of Coalitionally Bayesian Incentive Compatibility (CBIC).

Definition 4.1. An allocation $x=\left(x_{1}, \ldots, x_{n}\right) \in \bar{L}_{X}$, with or without free disposal, is said to be Transfer Coalitionally Bayesian Incentive Compatible (TCBIC) if it is not true that there exists a coalition $S$, states $\omega^{*}$ and $\omega^{\prime}$, with $\omega^{*}$ different from $\omega^{\prime}$ and $\omega^{\prime} \in \bigcap_{i \notin S} E_{i}\left(\omega^{*}\right)$ and a random net-trade vector, $z$, among the members of S,

$$
\left(z_{i}\right)_{i \in S}, \sum_{S} z_{i}=0
$$

such that for all $i \in S$ there exists $\bar{E}_{i}\left(\omega^{*}\right) \subseteq Z_{i}\left(\omega^{*}\right)=E_{i}\left(\omega^{*}\right) \cap\left(\bigcap_{j \notin S} E_{j}\left(\omega^{*}\right)\right)$, for which

$$
\begin{aligned}
& \sum_{\omega \in \bar{E}_{i}\left(\omega^{*}\right)} u_{i}\left(\omega, e_{i}(\omega)+x_{i}\left(\omega^{\prime}\right)-e_{i}\left(\omega^{\prime}\right)+z_{i}\right) q_{i}\left(\omega \mid \bar{E}_{i}\left(\omega^{*}\right)\right) \\
> & \sum_{\omega \in \bar{E}_{i}\left(\omega^{*}\right)} u_{i}\left(\omega, x_{i}(\omega)\right) q_{i}\left(\omega \mid \bar{E}_{i}\left(\omega^{*}\right)\right) .
\end{aligned}
$$

Notice that the $z_{i}$ 's above are not necessarily measurable. The definition is cast in terms of all possible $z_{i}^{\prime}$ s. It follows that $e_{i}(\omega)+x_{i}\left(\omega^{\prime}\right)-e_{i}\left(\omega^{\prime}\right)+z_{i}(\omega) \in X_{i}(\omega)$ is not necessarily measurable. The definition means that no coalition can form with the possibility that by misreporting a state, every member will become better off if the announcement is believed by the members of the complementary set.

Returning to Definition 4.1, one then can define CBIC to correspond to $z_{i}=0$ and then IBIC to the case when $S$ is a singleton. Thus we have (not IBCI) $\Rightarrow$ (not $\mathrm{CBIC}) \Rightarrow$ (not TCBIC). It follows that TCBIC $\Rightarrow \mathrm{CBIC} \Rightarrow$ IBIC.

We now provide a characterization of TCBIC:

10 See Krasa and Yannelis (1994) and Hahn and Yannelis (1997) for related concepts. 
Proposition 4.1. Let $\mathcal{E}$ be a one-good differential information economy as described above, and suppose each agent's utility function, $u_{i}=u_{i}\left(\omega, x_{i}(\omega)\right)$ is monotone in the elements of the vector of goods $x_{i}$, that $u_{i}\left(., x_{i}\right)$ is $\mathcal{F}_{i}$-measurable in the first argument, and that an element $x=\left(x_{1}, \ldots, x_{n}\right) \in \bar{L}_{X}^{1}$ is a feasible allocation in the sense that $\sum_{i=1}^{n} x_{i}(\omega)=\sum_{i=1}^{n} e_{i}(\omega) \forall \omega$. Consider the following conditions:

(i) $x \in L_{X}^{1}=\prod_{i=1}^{n} L_{X_{i}}^{1}$ and

(ii) $x$ is TCBIC.

Then (i) is equivalent to (ii).

Proof. First we show that (i) implies (ii) by showing that (i) and the negation of (ii) lead to a contradiction.

Let $x \in L_{X}$ and suppose that it is not TCBIC. Then, varying the notation for states to emphasize that Definition 4.1 does not hold, there exists a coalition $S$, states $a$ and $b$, with $a \neq b$ and $b \in \bigcap_{i \neq S} E_{i}(a)$ and a net-trade vector, $z$, among the members of $S$,

$$
\left(z_{i}\right)_{i \in S}, \quad \sum_{S} z_{i}=0
$$

such that for all $i \in S$ there exists $\bar{E}_{i}(a) \subseteq Z_{i}(a)=E_{i}(a) \cap\left(\bigcap_{j \notin S} E_{j}(a)\right)$, for which

$$
\begin{aligned}
& \sum_{c \in \bar{E}_{i}(\alpha)} u_{i}\left(c, e_{i}(c)+x_{i}(b)-e_{i}(b)+z_{i}\right) q_{i}\left(c \mid \bar{E}_{i}(a)\right) \\
> & \sum_{c \in \bar{E}_{i}(a)} u_{i}\left(c, x_{i}(c)\right) q_{i}\left(c \mid \bar{E}_{i}(a)\right) .
\end{aligned}
$$

For $c \in \bar{E}_{i}(a), e_{i}(c)=e_{i}(a)$ since $e_{i}$ is $\mathcal{F}_{i}$-measurable, so

$$
e_{i}(c)+x_{i}(b)-e_{i}(b)+z_{i}=e_{i}(a)+x_{i}(b)-e_{i}(b)+z_{i}
$$

and hence also

$$
u_{i}\left(c, e_{i}(c)+x_{i}(b)-e_{i}(b)+z_{i}\right)=u_{i}\left(a, e_{i}(a)+x_{i}(b)-e_{i}(b)+z_{i}\right),
$$

by the assumed $\mathcal{F}_{i}$-measurability of $u_{i}$.

Since, by (i), $x_{i}(c)=x_{i}(a)$ for $c \in \bar{E}_{i}(a)$, we similarly have $u_{i}\left(c, x_{i}(c)\right)=$ $u_{i}\left(a, x_{i}(a)\right)$. Thus in equation (7) the common utility terms can be lifted outside the summations giving

$$
u_{i}\left(a, e_{i}(a)+x_{i}(b)-e_{i}(b)+z_{i}\right)>u_{i}\left(a, x_{i}(a)\right)
$$

and hence $e_{i}(a)+x_{i}(b)-e_{i}(b)+z_{i}>x_{i}(a)$, by monotonicity of $u_{i}$.

Consequently,

$$
\sum_{i \in S}\left(x_{i}(b)-e_{i}(b)\right)>\sum_{i \in S}\left(x_{i}(a)-e_{i}(a)\right) .
$$


On the other hand for $i \notin S$ we have $x_{i}(b)-e_{i}(b)=x_{i}(a)-e_{i}(a)$ from which we obtain

$$
\sum_{i \notin S}\left(x_{i}(b)-e_{i}(b)\right)=\sum_{i \notin S}\left(x_{i}(a)-e_{i}(a)\right) .
$$

Taking equations (8),(9) together we have

$$
\sum_{i \in I}\left(x_{i}(b)-e_{i}(b)\right)>\sum_{i \in I}\left(x_{i}(a)-e_{i}(a)\right),
$$

which is a contradiction since both sides are equal to zero, by feasibility. ${ }^{11}$

We now show that (ii) implies (i). For suppose not. Then there exists some Agent $\mathrm{j}$ and states $a, b$ with $b \in E_{j}(a)$ such that $x_{j}(a) \neq x_{j}(b)$. Without loss of generality, we may assume that $x_{j}(a)>x_{j}(b)$. Since $e_{j}($.$) is \mathcal{F}_{j}$-measurable $e_{j}(b)=e_{j}(a)$ and therefore

$$
x_{j}(a)-e_{j}(a)>x_{j}(b)-e_{j}(b) .
$$

Let $S=I \backslash\{j\}$. From the feasibility of $x$ and (11) it follows that

$$
\begin{aligned}
\sum_{i \in S}\left(x_{i}(a)-e_{i}(a)\right) & =-\left(x_{j}(a)-e_{j}(a)\right)<-\left(x_{j}(b)-e_{j}(b)\right) \\
& =\sum_{i \in S}\left(x_{i}(b)-e_{i}(b)\right) .
\end{aligned}
$$

From (12) we have that

$$
\delta=\sum_{i \in S}\left(e_{i}(a)+x_{i}(b)-e_{i}(b)-x_{i}(a)\right)>0 .
$$

For each $i \in S$, let

$$
z_{i}=x_{i}(a)-e_{i}(a)-x_{i}(b)+e_{i}(b)+\frac{\delta}{n-1}
$$

so that $\sum_{i \in S} z_{i}=0$ and

$$
e_{i}(a)+x_{i}(b)-e_{i}(b)+z_{i}>x_{i}(a) .
$$

By monotonicity of $u_{i}$, we can conclude that

$$
u_{i}\left(a, e_{i}(a)+x_{i}(b)-e_{i}(b)+z_{i}\right)>u_{i}\left(a, x_{i}(a)\right),
$$

for all $i \in S$, a contradiction to the fact that $x$ is TCBIC as the role of $\bar{E}_{i}$ in the definition can be played by $\{a\}$.

Finally note that a particular case of $\mathcal{F}_{i}$-measurability of $u_{i}$ is when it is independent of $\omega$. This completes the proof of Proposition 4.1.

11 Koutsougeras and Yannelis (1993) and Krasa and Yannelis (1994) show that (i) implies (ii) for any number of goods, but for ex post utility functions. This means that the contract is made ex ante and after the state of nature is realized we see that we have incentive compatibility. Hahn and Yannelis (1997) show that (i) implies (ii) for any number of goods and for interim utility functions. Notice that since the non-free disposal Radner equilibrium is a subset of the non-free disposal ex ante private core, it follows from Hahn and Yannelis that the non-free disposal Radner equilibrium is TCBIC. 
In the lemma that follows we refer to CBIC, as TCBIC does not make much sense since $z_{i}$ is not available. CBIC is obtained when all $z_{i}$ 's are set equal to zero.

Lemma 4.1. Under the conditions of the Proposition, if there are only two agents then (ii) $x$ is CBIC, which is the same as IBIC, implies (i).

Proof. For suppose not. Then lack of $\mathcal{F}_{i}$-measurability of the allocations implies that there exist Agent $j$ and states a, b, where $b \in E_{j}(a)$, such that $x_{j}(b)<x_{j}(a)$ and therefore

$$
x_{j}(b)-e_{j}(b)<x_{j}(a)-e_{j}(a) .
$$

Feasibility implies

$$
x_{i}(b)-e_{i}(b)+x_{j}(b)-e_{j}(b)=x_{i}(a)-e_{i}(a)+x_{j}(a)-e_{j}(a)
$$

from which we obtain

$$
x_{i}(b)-e_{i}(b)>x_{i}(a)-e_{i}(a) .
$$

By monotonicity and the one-good per state assumption it follows that,

$$
u_{i}\left(a, e_{i}(a)+x_{i}(b)-e_{i}(b)\right)>u_{i}\left(a, x_{i}(a)\right) .
$$

This implies that we have

$$
u_{i}\left(a, e_{i}(c)+x_{i}(b)-e_{i}(b)\right)>u_{i}\left(a, x_{i}(c)\right)
$$

which contradicts the assumption that $x$ is CBIC. This completes the proof of the lemma.

The above results characterize TCBIC and CBIC in terms of private individual measurability, i.e. $\mathcal{F}_{i}$-measurability, of allocations. These results will enable us to conclude whether or not, in case of non-free disposal, any of the solution concepts, i.e. Radner equilibrium, REE, private core, weak fine core and weak fine value will be TCBIC whenever feasible allocations are $\mathcal{F}_{i}$-measurable.

It follows from the lemma that the redistribution shown in the matrix below, which is a weak fine core allocation of Example 3.2, where the ith line refers to Player $\mathrm{i}$ and the columns from left to right to states $a, b$ and $c$,

$$
\left(\begin{array}{lll}
5 & 2.5 & 2.5 \\
5 & 2.5 & 2.5
\end{array}\right)
$$

is not $\mathrm{CBIC}$ as it is not $\mathcal{F}_{i}$-measurable. Thus, a weak fine core allocation may not be $\mathrm{CBIC}$.

On the other hand the proposition implies that, in Example 3.2, the no-trade allocation

$$
\left(\begin{array}{lll}
5 & 5 & 0 \\
5 & 0 & 5
\end{array}\right)
$$

is incentive compatible. This is a non-free disposal REE, and a private core allocation. 
We note that the Proposition 4.1 refers to non-free disposal. As a matter of fact Proposition 4.1 is not true if we assume free disposal. Indeed if free disposal is allowed $\mathcal{F}_{i}$-measurability PBE does not imply incentive compatibility.

In the case with free disposal, private core and Radner equilibrium need not be incentive compatible. In order to see this we notice that in Example 3.2 the (free disposal) Radner equilibrium is $x_{1}=(4,4,1)$ and $x_{2}=(4,1,4)$. The above allocation is clearly $\mathcal{F}_{i}$-measurable and it can easily be checked that it belongs to the (free disposal) private core. However it is not TBIC since if state a occurs Agent 1 has an incentive to report state $\mathrm{c}$ and gain.

Now in employing game trees in the analysis, as it is done below, we will adopt the definition of IBIC. The equilibrium concept employed will be that of PBE. The definition of a play of the game is a directed path from the initial to a terminal node.

In terms of the game trees, a core allocation will be IBIC if there is a profile of optimal behavioral strategies and equilibrium paths along which no player misreports the state of nature he has observed. This allows for the possibility, as we shall see later, that such strategies could imply that players have an incentive to lie from information sets which are not visited by an optimal play.

In view of the analysis in terms of game trees we comment again on the general idea of CBIC. First we look at it once more, in a similar manner to the one in the beginning of Section 4.

Suppose the true state of nature is $\bar{\omega}$. Any coalition can only see that the state lies in $\bigcap_{i \in S} E_{i}(\bar{\omega})$ when they pool their observations. If they decide to lie they must first guess at what is the true state and they will do so at some $\omega^{*} \in \bigcap_{i \in S} E_{i}(\bar{\omega})$. Then of course we have $\bigcap_{i \in S} E_{i}(\bar{\omega})=\bigcap_{i \in S} E_{i}\left(\omega^{*}\right)$. Having decided on $\omega^{*}$ as a possible true state, they now pick some $\omega^{\prime} \in \bigcap_{j \notin S} E_{j}\left(\omega^{*}\right)$ and (assuming the system is not CBIC) they hope, by announcing (each of them) that they have seen $E_{i}\left(\omega^{\prime}\right)$ to secure better payoffs.

This is all contingent on their being believed by $I \backslash S$. This, in turn, depends on their having been correct in their guessing that $\omega^{*}=\bar{\omega}$, in which case they might be believed. If $\omega^{*} \neq \bar{\omega}$, i.e they guess wrongly, then since $\bigcap_{j \notin S} E_{j}\left(\omega^{*}\right) \neq \bigcap_{j \notin S} E_{j}(\bar{\omega})$ they may be detected in their lie, since possibly $\omega^{\prime} \notin \bigcap_{j \notin S} E_{j}(\bar{\omega})$.

This is why the definition of CBIC can only be about possible existence of situations where a lie might be beneficial. It is not concerned with what happens if the lie is detected. On the other hand the extensive form forces us to consider that alternative. It requires statements concerning earlier decisions by other players to lie or tell the truth and what payoffs will occur whenever a lie is detected, through observations or incompatibility of declarations. Only in this fuller description can players really make a decision whether to risk a lie, since only then can they balance the gains from not being caught against a definitely declared payoff if they are.

The issue is whether cooperative and noncooperative static solutions can be obtained as perfect Bayesian or sequential equilibria. That is whether such allocations can also be supported through an appropriate noncooperative solution concept. The 
analysis below shows that CBIC allocations can be supported by a PBE while lack of incentive compatibility implies non-support, in the sense that the two agents, left on their own, do not sign the contract. It is also shown how implementation of allocations becomes possible through the introduction in the analysis of an exogenous third party or an endogenous intermediary.

\section{Non-implementation of free disposal private core and Radner equilibria, and of weak fine core allocations}

The main point here is that lack of IBIC implies that the two agents based on their information cannot sign a proposed contract because both of them have an incentive to cheat the other one and benefit. Indeed PBE leads to no-trade. This so irrespective of whether in state $a$ the contract specifies that they both get 5 or 4 .

Note that to impose free disposal in state $a$ causes certain problems, because the question arises as to who will check that the agents have actually thrown away 1 unit. In general, free disposal is not always a very satisfactory assumption in differential information economies with monotone preferences.

We shall investigate the possible implementation of the allocation

$$
\left(\begin{array}{lll}
4 & 4 & 1 \\
4 & 1 & 4
\end{array}\right)
$$

in Example 3.2, contained in a contract between P1 and P2 when no third party is present. For the case with free disposal, this is both a private core and a Radner equilibrium allocation.

This allocation is not IBIC because, as we explained in the previous section, if Agent 1 observes $\{a, b\}$, he has an incentive to report $c$ and Agent 2 has an incentive to report $b$ when he observes $\{a, c\}$.

We construct a game tree and employ reasonable rules for describing the outcomes of combinations of states of nature and actions of the players. In fact we look at the contract

$$
\left(\begin{array}{lll}
5 & 4 & 1 \\
5 & 1 & 4
\end{array}\right)
$$

in which the agents get as much per state as under the private core allocation above. The latter can be obtained by invoking free disposal in state $a$.

The investigation is through the analysis of a specific sequence of decisions and information sets shown in the game tree in Figure 1. Notice that vectors at the terminal nodes of a game tree will refer to payoffs of the players in terms of quantities. The first element will be the payoff to P1, etc.

The players are given strategies to tell the truth or to lie, i.e., we model the idea that agents truly inform each other about what states of nature they observe, or deliberately aim to mislead their opponent. The issue is what type of behavior is optimal and therefore whether a proposed contract will be signed or not. We find that the optimal strategies of the players imply no-trade.

Figures 1 and 2 show that the allocation $(5,4,1)$ and $(5,1,4)$ will be rejected by the players. They prefer to stay with their initial endowments and will not sign the proposed contract as it offers to them no advantage. 


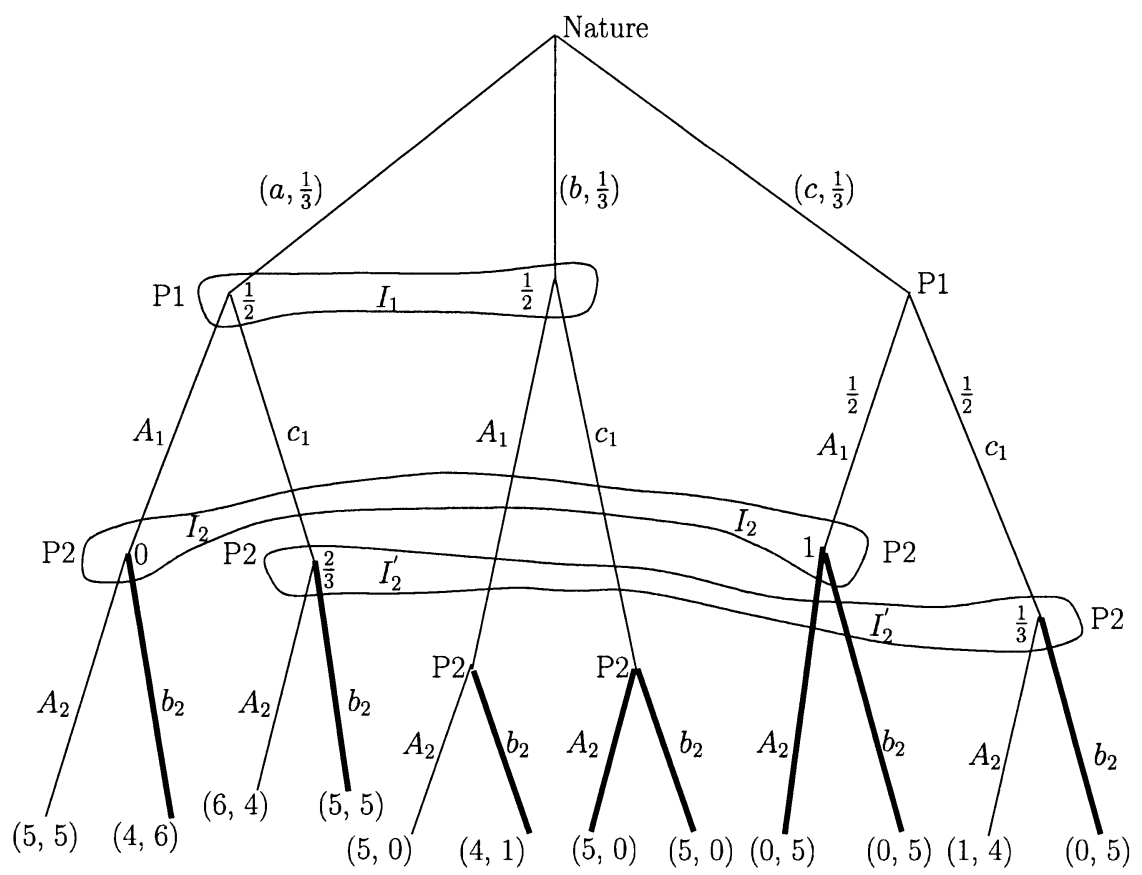

Figure 1

In Figure 1, nature chooses states $a, b$ or $c$ with equal probabilities. This choice is flashed on a screen which both players can see. P1 cannot distinguish between $a$ and $b$, and $\mathrm{P} 2$ between $a$ and $c$. This accounts for the information sets $I_{1}, I_{2}$ and $I_{2}^{\prime}$ which have more than one node. A player to which such an information set belongs cannot distinguish between these nodes and therefore his decisions are common to all of them. A behavioral strategy of a player is to declare which choices he would make, with what probability, from each of his information sets. Indistinguishable nodes imply the $\mathcal{F}_{i}$-measurability of decisions.

P1 moves first and he can either play $A_{1}=\{a, b\}$ or $c_{1}=\{c\}$, i.e., he can say "I have seen $\{a, b\}$ or "I have seen $c$ ". Of course only one of these declarations will be true. Then $\mathrm{P} 2$ is to respond saying that the signal he has seen on the screen is $A_{2}=\{a, c\}$ or that it is $b_{2}=\{b\}$. Obviously only one of these statements is true.

Strictly speaking the notation for choices should vary with the information set but there is no danger of confusion here. Finally notice that the structure of the game tree is such that when P2 is to act he knows exactly what P1 has chosen.

Next we specify the rules for calculating the payoffs, i.e. the terms of the contract:

(i) If the declarations by the two players are incompatible, that is $\left(c_{1}, b_{2}\right)$ then no-trade takes place and the players retain their initial endowments. That is the case when either state $\mathrm{c}$, or state $\mathrm{b}$ occurs and Agent 1 reports state $\mathrm{c}$ and Agent 2 state $b$. In state a both agents can lie and the lie cannot be detected by either of them. They are in the events $\{a, b\}$ and $\{a, c\}$ respectively, they get 


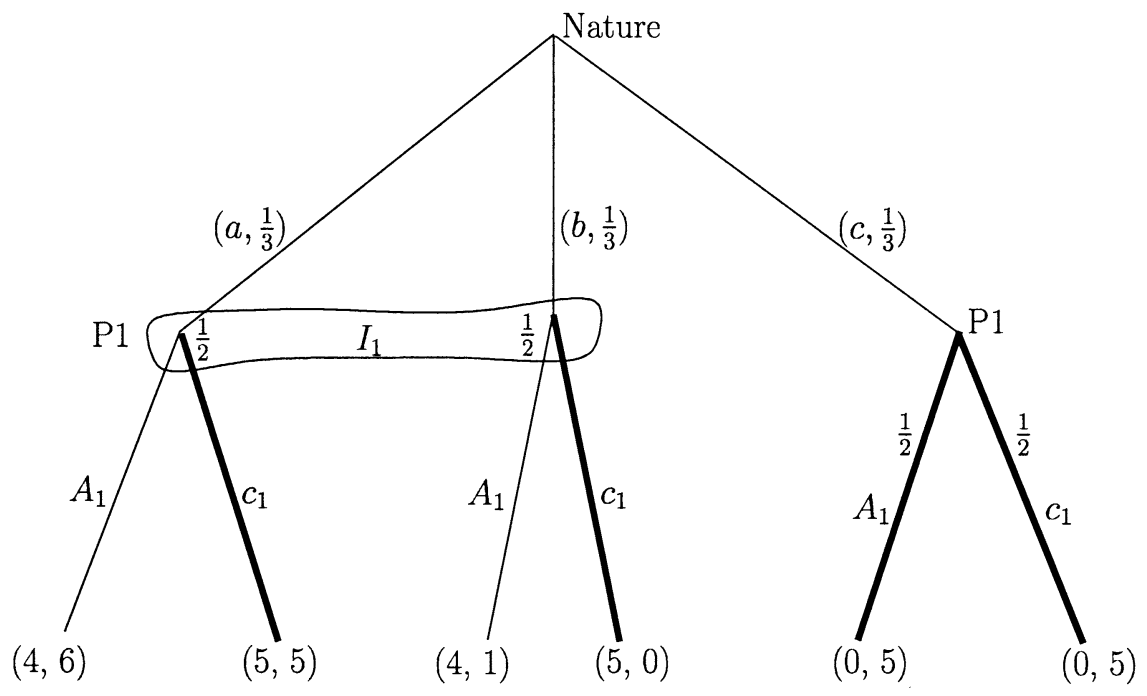

Figure 2

5 units of the initial endowments and again they are not willing to cooperate. Therefore whenever the declarations are incompatible, no trade takes place and the players retain their initial endowments.

(ii) If the declarations are $\left(A_{1}, A_{2}\right)$ then even if one of the players is lying, this cannot be detected by his opponent who believes that state $a$ has occured and both players have received endowment 5 . Hence no-trade takes place.

(iii) If the declarations are $\left(A_{1}, b_{2}\right)$ then a lie can be beneficial and undetected. P1 is trapped and must hand over one unit of his endowment to P2. Obviously if his initial endowment is zero then he has nothing to give.

(iv) If the declarations are $\left(c_{1}, A_{2}\right)$ then again a lie can be beneficial and undetected. P2 is now trapped and must hand over one unit of his endowment to P1. Obviously if his initial endowment is zero then he has nothing to give.

The calculations of payoffs do not require the revelation of the actual state of nature. Optimal decisions will be denoted by a heavy line. We could assume that a player does not lie if he cannot get a higher payoff by doing so.

Assuming that each player chooses optimally from his information sets, the game in Figure 1 folds back to the one in Figure 2. Inspection of Figure 1 reveals that from the information set $I_{2}$ agent P2 can play $b_{2}$ with probability 1 . (A heavy line $A_{2}$ indicates that this choice also would not affect the analysis). This accounts for the payoff $(4,6)$ and the first payoff $(0,5)$ from left to right in Figure 2 . Similarly by considering the optimal decisions from all other information sets of $\mathrm{P} 2$ we arrive at Figure 2. Analyzing this figure we obtain the optimal strategies of P1.

In conclusion, the optimal behavioral strategy for $\mathrm{P} 1$ is to play $c_{1}$ with probability 1 from $I_{1}$, i.e to lie, and from the singleton to play any probability mixture of options, and we have chosen $\left(A_{1}, \frac{1}{2} ; c_{1}, \frac{1}{2}\right)$. The optimal strategy of $\mathrm{P} 2$ is to play $b_{2}$ from both $I_{2}$ and $I_{2}^{\prime}$, i.e. to lie, and from the second singleton he can either tell 
the truth or lie, or spin a wheel, divided in proportions corresponding to $A_{1}$ and $c_{1}$, to decide what to choose.

In Figures 1 and 2, the fractions next to the nodes in the information sets correspond to beliefs of the agents obtained, wherever possible, through Bayesian updating. I.e., they are consistent with the choice of a state by nature and the optimal behavioral strategies of the players. This means that strategies and beliefs satisfy the conditions of a PBE.

These probabilities are calculated as follows. From left to right, we denote the nodes in $I_{1}$ by $j_{1}$ and $j_{2}$, in $I_{2}$ by $n_{1}$ and $n_{2}$ and in $I_{2}^{\prime}$ by $n_{3}$ and $n_{4}$. Given the choices by nature, the strategies of the players described above and using the Bayesian formula for updating beliefs we can calculate, for example, the conditional probabilities

$$
\begin{aligned}
\operatorname{Pr}\left(n_{1} / A_{1}\right) & =\frac{\operatorname{Pr}\left(A_{1} / n_{1}\right) \times \operatorname{Pr}\left(n_{1}\right)}{\operatorname{Pr}\left(A_{1} / n_{1}\right) \times \operatorname{Pr}\left(n_{1}\right)+\operatorname{Pr}\left(A_{1} / n_{2}\right) \times \operatorname{Pr}\left(n_{2}\right)} \\
& =\frac{1 \times 0}{1 \times 0+1 \times \frac{1}{3} \times \frac{1}{2}}=0
\end{aligned}
$$

and

$$
\begin{aligned}
\operatorname{Pr}\left(n_{3} / c_{1}\right) & =\frac{\operatorname{Pr}\left(c_{1} / n_{3}\right) \times \operatorname{Pr}\left(n_{3}\right)}{\operatorname{Pr}\left(c_{1} / n_{3}\right) \times \operatorname{Pr}\left(n_{3}\right)+\operatorname{Pr}\left(c_{1} / n_{4}\right) \times \operatorname{Pr}\left(n_{4}\right)} \\
& =\frac{1 \times \frac{1}{3}}{1 \times \frac{1}{3}+1 \times \frac{1}{2} \times \frac{1}{3}}=\frac{2}{3} .
\end{aligned}
$$

In Figure 3 we indicate, through heavy lines, plays of the game which are the outcome of the choices by nature and the optimal behavioral strategies by the players. The interrupted heavy lines signify that nature does not take an optimal decision but simply chooses among three alternatives, with equal probabilities. The directed path $\left(a, c_{1}, b_{2}\right)$ with payoffs $(5,5)$ occurs with probability $\frac{1}{3}$. The paths $\left(b, c_{1}, A_{2}\right)$ and $\left(b, c_{1}, b_{2}\right)$ lead to payoffs $(5,0)$ and occur with probability $\frac{1}{3}(1-q)$ and $\frac{1}{3} q$, respectively. The values $(1-q)$ and $q$ denote the probabilities with which $\mathrm{P} 2$ chooses between $A_{2}$ and $b_{2}$ from the singleton node at the end of $\left(b, c_{1}\right)$. The paths $\left(c, A_{1}, b_{2}\right)\left(c, c_{1}, b_{2}\right)$ lead to payoffs $(0,5)$ and occur, each, with probability $\frac{1}{3} \times \frac{1}{2}$.

For all choices by nature, at least one of the players tells a lie on the optimal play. The players by lying avoid the possibility of having to make a payment to their opponent and stay with their initial endowments. The PBE obtained above confirms the initial endowments. The decisions to lie imply that the players will not sign the contract $(5,4,1)$ and $(5,1,4)$.

We have constructed an extensive form game and employed reasonable rules for calculating payoffs and shown that the proposed allocation $(5,4,1)$ and $(5$, $1,4)$ will not be realized. A similar conclusion would have been reached if we investigated the allocation $(4,4,1)$ and $(4,1,4)$ which would have been brought about by considering free disposal.

Finally suppose we were to modify (iii) and (iv) of the rules and adopt those in Section 5 of Glycopantis, Muir, and Yannelis (2001): 


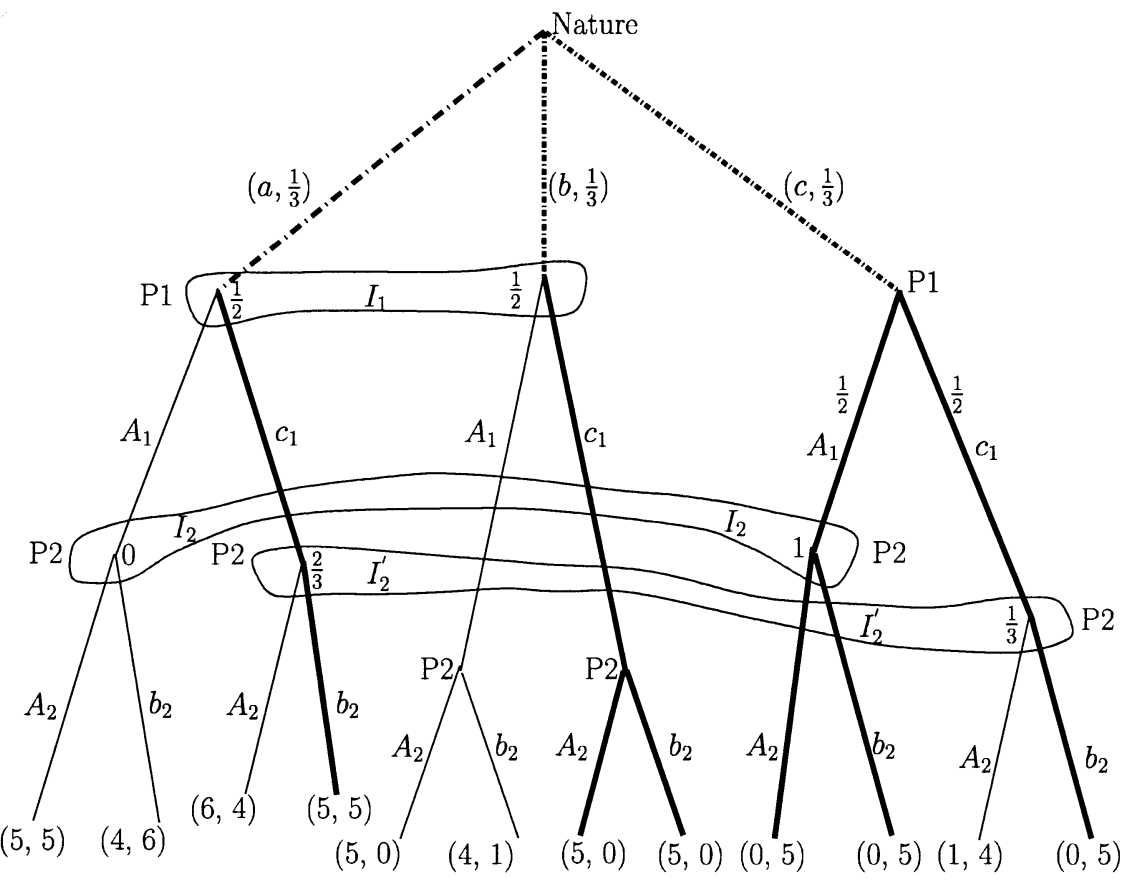

Figure 3

(iii) If the declarations are $\left(A_{1}, b_{2}\right)$ then a lie can be beneficial and undetected, and $\mathrm{P} 1$ is trapped and must hand over half of his endowment to P2. Obviously if his endowment is zero then he has nothing to give.

(iv) If the declarations are $\left(c_{1}, A_{2}\right)$ then again a lie can be beneficial and undetected. $\mathrm{P} 2$ is now trapped and must hand over half of his endowment to P1. Obviously if his endowment is zero then he has nothing to give.

The new rules would imply, starting from left to right, the following changes in the payoffs in Figure 1. The second vector would now be $(2.5,7.5)$, the third vector $(7.5,2.5)$, the sixth vector $(2.5,2.5)$ and the eleventh vector $(2.5,2.5)$. The analysis in Glycopantis, Muir, and Yannelis (2001) shows that the weak fine core allocation in which both agents receive $(5,2.5,2.5)$ cannot be implemented as a PBE. Again this allocation is not IBIC.

Since we have two agents, the weak fine value belongs to the weak fine core. We can also check through routine calculations that the non-implementable allocation $x_{1}=x_{2}=(5,2.5,2.5)$ belongs to the weak fine value, with the two agents receiving equal weights.

Finally we note that, in the context of Figure 1, the perfect Bayesian equilibrium implements the initial endowments allocation

$$
\left(\begin{array}{lll}
5 & 5 & 0 \\
5 & 0 & 5
\end{array}\right) .
$$


In the case of non-free disposal, no-trade coincides with the REE and it is implementable. However as it is shown in Glycopantis, Muir, and Yannelis (2002) a REE is not in general implementable.

\section{Implementation of private core and Radner equilibria through the courts; implementation of weak fine core}

We shall show here how the free disposal private core and also Radner equilibrium allocation

$$
\left(\begin{array}{lll}
4 & 4 & 1 \\
4 & 1 & 4
\end{array}\right)
$$

of Example 3.2 can be implemented as a PBE by invoking an exogenous third party, which can be interpreted as a court which imposes penalties when the agents lie.

We shall assume that the agents do not hear the choice announced by the other player or that they do not pay much attention to each other because the court will verify the true state of nature.

It should be noted that now if the two players see the events $\left(A_{1}, A_{2}\right)$ the exogenous agent will not allow them to misreport the state of nature by imposing a penalty for lying. Therefore the contract will be enforced exogenously.

The analysis is through the figures below. Figure 4 contains the information sets of the two agents, P1 and P2, their sequential decisions and the payoffs in terms of quantities. Each agent can choose either to tell the truth about the information set he is in, or to lie.

Nature chooses states $a, b$ and $c$ with equal probabilities. P1 acts first and cannot distinguish between $a$ and $b$. When P2 is to act he has two kinds of ignorance. Not only he cannot distinguish between $a$ and $c$ but also he does not know what P1 has chosen before him. This is an assumption about the relation between decisions. The one unit that the courts take from a lying agent can be considered to cover the costs of the court.

Next given the sequence of decisions of the two players, shown on the tree, we specify the rules for calculating payoffs in terms of quantities, i.e we specify the terms of the contract. They will, of course include the penalties that the court would impose to the agents for lying.

The rules are:

(i) If a player lies about his observation, then he is penalized by 1 unit of the good. If both players lie then they are both penalized. For example if the declarations are $\left(c_{1}, b_{2}\right)$ and state $a$ occurs both are penalized. If they choose $\left(c_{1}, A_{2}\right)$ and state $a$ occurs then the first player is penalized. If a player lies and the other agent has a positive endowment then the court keeps the quantity substracted for itself. However, if the other agent has no endowment, then the court transfers to him the one unit subtracted from the one who lied.

(ii) If the declarations of the two agents are consistent, that is $\left(A_{1}, A_{2}\right)$ and state $a$ occurs, $\left(A_{1}, b_{2}\right)$ and state $b$ occurs, $\left(c_{1}, A_{2}\right)$ and state $c$ occurs, then they divide equally the total endowments in the economy. 


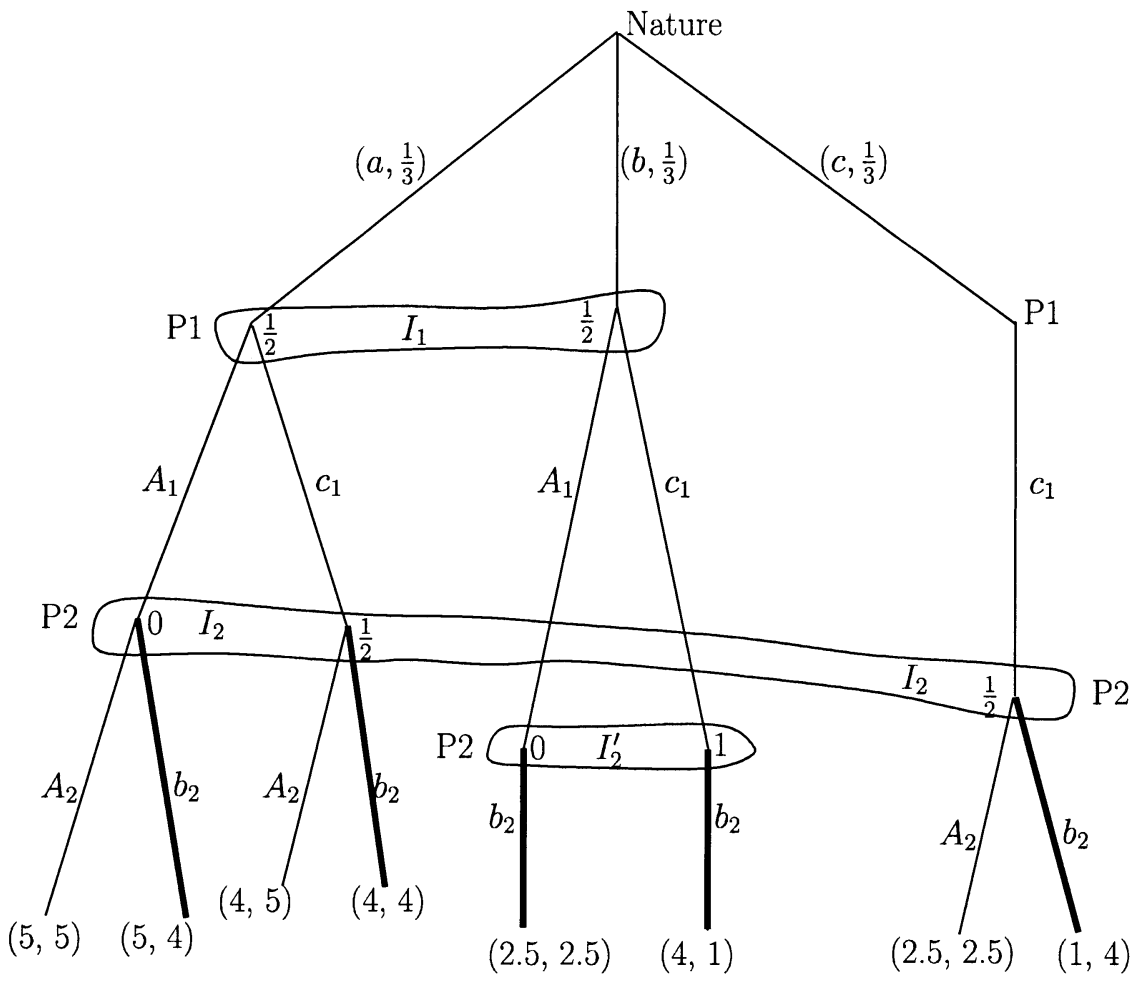

Figure 4

One explanation of the size of the payoffs is that if the agents decide to share, they do so voluntarily. On the other hand the court feel that they can punish them for lying but not to the extent of forcing them to share their endowments.

Assuming that each player chooses optimally, given his stated beliefs, from the information sets which belong to him, P2 chooses to play $b_{2}$ with probability 1 from both $I_{2}$ and $I_{2}^{\prime}$ and the game in Figure 4 folds back to the one in Figure 5. The choice of $b_{2}$ is justified as follows. We ignore for the moment the specific conditional probabilities attached to the nodes of $I_{2}$. On the other hand, starting from left to right, the sum of the probabilities of the first two nodes must be equal to $\frac{1}{2}$, and this implies that strategy $b_{2}$ overtakes, in utility terms, strategy $A_{2}$, as $\frac{1}{2} 5^{\frac{1}{2}}+\frac{1}{2} 2.5^{\frac{1}{2}}<4^{\frac{1}{2}}$. It follows that $\mathrm{P} 2$ chooses to play the behavioral strategy $b_{2}$ with probability 1 . Now inspection of Figure 5 implies that P1 will choose $c_{1}$ from $I_{1}$. The conditional probabilities on the nodes of $I_{1}$ follow from the fact that nature chooses with equal probabilities and the optimal choice of $c_{1}$ with probability 1 follows again from the fact that $\frac{1}{2} 5^{\frac{1}{2}}+\frac{1}{2} 2.5^{\frac{1}{2}}<4^{\frac{1}{2}}$.

Figure 6 indicates, through heavy lines, plays of the game which are the outcome of choices by nature and the optimal strategies of the players. The fractions next to the nodes of the information sets are obtained through Bayesian updating. I.e. 


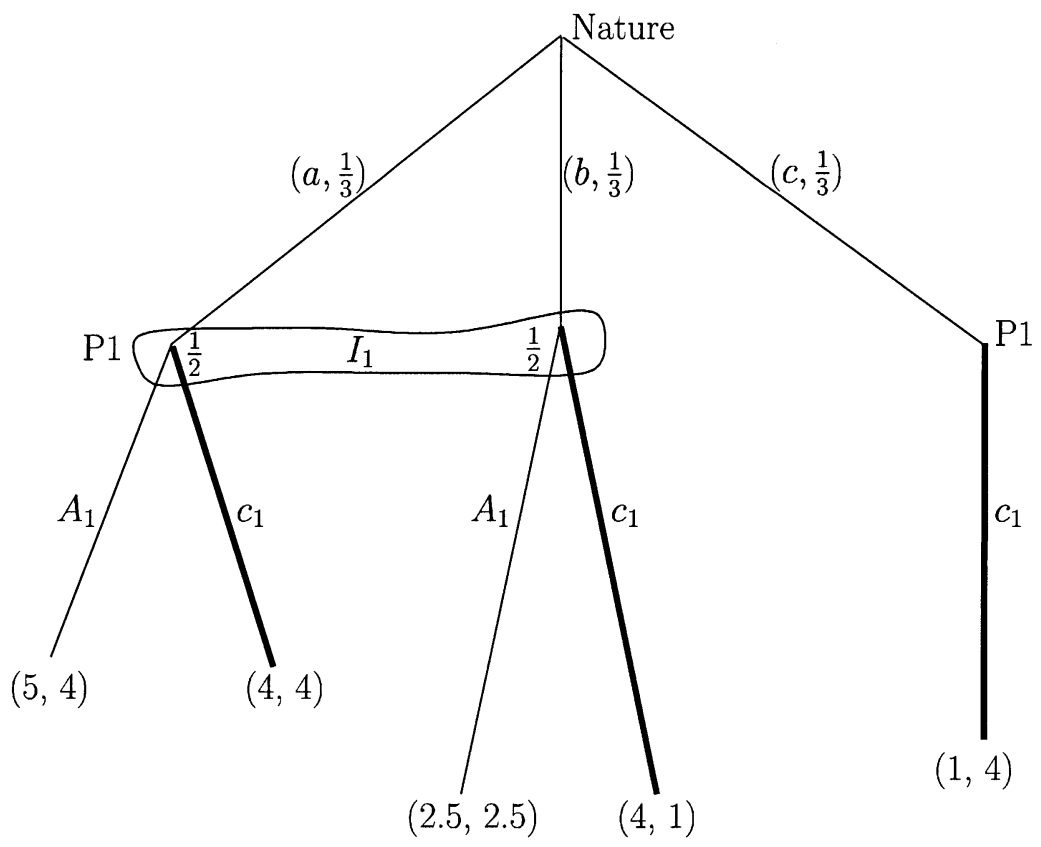

Figure 5

they are consistent with the choice of a state by nature and the optimal behavioral strategies of the players. We have thus obtained a PBE and the above argument implies that it is unique.

The free disposal private core allocation that we are concerned with is implemented, always, by at least one of the agents lying. The reason is that they make the same move from all the nodes of an information set and the rules of the game imply that they are not eager to share their endowments. They prefer to suffer the penalty of the court.

Finally notice the following. Suppose that the penalties are changed as follows. The court is extremely severe when an agent lies while the other agent has no endowment. It takes all the endowment from the one who is lying and transfers it to the other player. Everything else stays the same. Then the game is summarized in a modified Figure 4 . Numbering the end points from left to right, the 2 nd vector will be replaced by $(5,0)$, the 3 rd by $(0,5)$, the 4 th by $(0,0)$, the 6 th by $(0,5)$ and the 8 th one by $(5,0)$.

The analysis of the game implies now that $\mathrm{P} 2$ will play $A_{2}$ from $I_{2}$ and $\mathrm{P} 1$ will play $A_{1}$ from $I_{1}$. Therefore invoking an exogenous agent implies that the PBE will now implement the weak fine core allocation

$$
\left(\begin{array}{lll}
5 & 2.5 & 2.5 \\
5 & 2.5 & 2.5
\end{array}\right)
$$




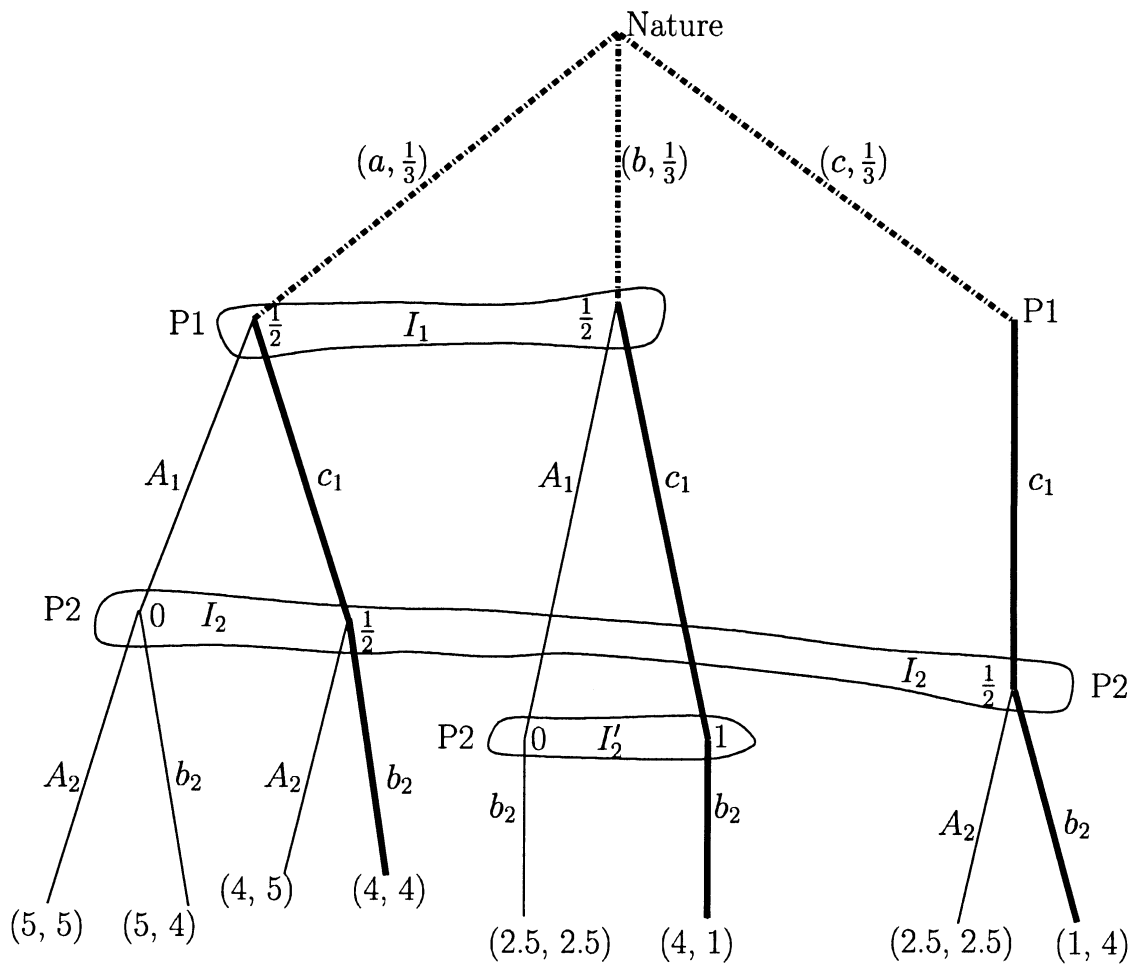

Figure 6

\section{Implementation of non-free disposal private core through an endogenous intermediary}

Here we draw upon the discussion in Glycopantis, Muir, and Yannelis (2001) but we add the analysis that the optimal paths obtained are also part of a sequential equilibrium. Hence we obtain a stronger conclusion, in the sense that we implement the private core allocation as a sequential equilibrium, which requires more conditions than PBE.

In the case we consider now there is no court and the agents in order to decide must listen to the choices of the other players before them. The third agent, P3, is endogenous and we investigate his role in the implementation, or realization, of private core allocations.

Private core without free disposal seems to be the most satisfactory concept. The third agent who plays the role of the intermediary implements the contract and gets rewarded in state $a$. We shall consider the private core allocation, of Example 3.1,

$$
\left(\begin{array}{lll}
4 & 4 & 1 \\
4 & 1 & 4 \\
2 & 0 & 0
\end{array}\right) .
$$


We know that such core allocations are CBIC and we shall show now how they can be supported as a perfect Bayesian equilibrium of a noncooperative game.

$\mathrm{P} 1$ cannot distinguish between states $a$ and $b$ and $\mathrm{P} 2$ between $a$ and $c$. P3 sees on the screen the correct state and moves first. He can either announce exactly what he saw or he can lie. Obviously he can lie in two ways. When P1 comes to decide he has his information from the screen and also he knows what P3 has played. When P2 comes to decide he has his information from the screen and he also knows what P3 and P1 played before him. Both P1 and P2 can either tell the truth about the information they received from the screen or they can lie.

We must distinguish between the announcements of the players and the true state of nature. The former, with the players' temptations to lie, cannot be used to determine the true state which is needed for the purpose of making payoffs. $\mathrm{P} 3$ has a special status but he must also take into account that eventually the lie will be detected and this can affect his payoff.

The rules of calculating payoffs, i.e. the terms of the contract, are as follows:

If $\mathrm{P} 3$ tells the truth we implement the redistribution in the matrix above which is proposed for this particular choice of nature.

If $\mathrm{P} 3$ lies then we look into the strategies of P1 and P2 and decide as follows:

(i) If the declaration of P1 and P2 are incompatible we go to the initial endowments and each player keeps his.

(ii) If the declarations are compatible we expect the players to honour their commitments for the state in the overlap, using the endowments of the true state, provided these are positive. If a player's endowment is zero then no transfer from that agent takes place as he has nothing to give.

The extensive form game is shown in Figure 7, in which the heavy lines can be ignored in the first instance. We are looking for a PBE, i.e. a set of optimal behavioral strategies consistent with a set of beliefs. The beliefs are indicated by the probabilities attached to the nodes of the information sets, with arbitrary $r, s, q, p$ and $t$ between 0 and 1 . The folding up of the game tree through optimal decisions by $\mathrm{P} 2$, then by $\mathrm{P} 1$ and subsequently by P3 is explained in Glycopantis, Muir, and Yannelis (2001).

In Figure 7 we indicate through heavy lines the equilibrium paths. The interrupted heavy lines at the beginning of the tree signify that nature does not take an optimal decision but simply chooses among three alternatives, with equal probabilities. The directed paths $\left(a, a, A_{1}, A_{2}\right)$ with payoffs $(4,4,2),\left(b, b, A_{1}, b_{2}\right)$ with payoffs $(4,1,0)$ and $\left(c, c, c_{1}, A_{2}\right)$ with payoffs $(1,4,0)$ occur, each, with probability $\frac{1}{3}$. It is clear that nobody lies on the optimal paths and that the proposed reallocation is incentive compatible and hence it will be realized.

Along the optimal paths nobody has an incentive to misrepresent the realized state of nature and hence the private core allocation is incentive compatible. However even optimal strategies can imply that players might have an incentive to lie from information sets which are not visited by the optimal play of the game. For example, $\mathrm{P} 1$, although he knows that nature has chosen $a$ or $b$, has an incentive to declare $c_{1}$ from $I_{1}^{3}$, trying to take advantage of a possible lie by P3. Similarly P2, although he knows that nature has chosen $a$ or $c$, has an incentive to declare $b_{2}$ from 


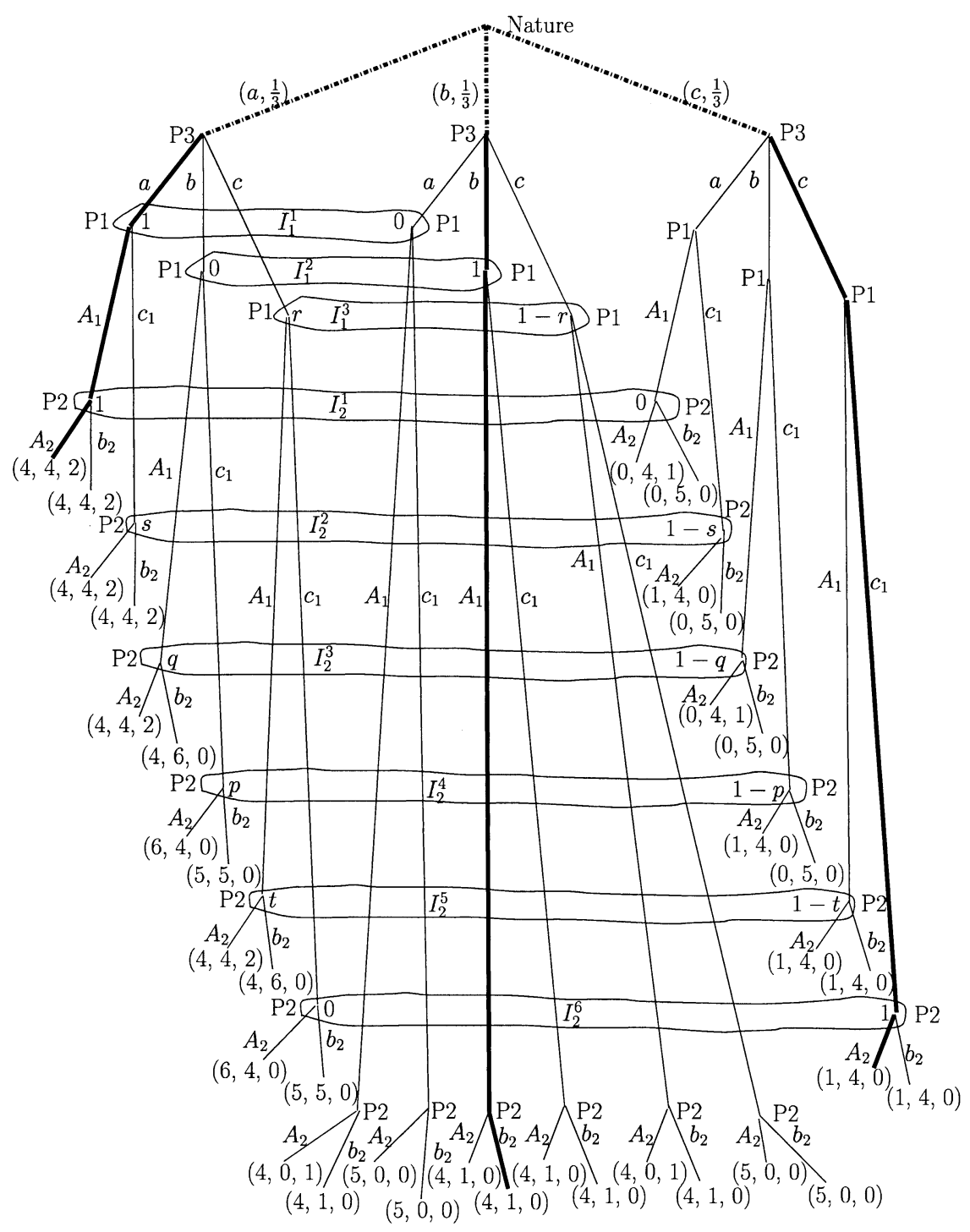

Figure 7

$I_{2}^{2}, I_{2}^{3}, I_{2}^{4}$ and $I_{2}^{5}$, trying to take advantage of possible lies by the other players. Incentive compatibility has now been defined to allow that the optimal strategies can contain lies, while there must be an optimal play which does not.

We also note that the same payoffs, i.e. $(4,4,2),(4,1,0)$ and $(1,4,0)$, can be confirmed as a PBE for all possible orders of the players.

Next we turn our attention to obtaining a sequential equilibrium. This adds further conditions to those of a PBE. Now, it is also required that the optimal behavioral strategies and the beliefs consistent with these are the limit of a sequence 
consisting of completely stochastic behavioral strategies, that is all choices are played with positive probability, and the implied beliefs. Throughout the sequence it is only required that beliefs are consistent with the strategies. The latter are not expected to be optimal.

We discuss how the PBE shown in Figure 7 can also be obtained as a sequential equilibrium in the sense of Kreps and Wilson (1982). Therefore, we are looking for a sequence of positive probabilities attached to all the choices from each information set and beliefs consistent with these such that their limits are the results given in Figure 7.

First we specify the positive probabilities, i.e. the completely stochastic strategies, with which the players choose the available actions. The sequence is obtained through $\{n=2,3, \ldots\}$.

In the first instance we consider the singletons from left to right belonging to $\mathrm{P} 3$. At the first one the positive probabilities attached to the various actions are given by $\left(a, 1-\frac{2}{n} ; b, \frac{1}{n} ; c, \frac{1}{n}\right)$, at the second one by $\left(a, \frac{1}{n} ; b, 1-\frac{2}{n} ; c, \frac{1}{n}\right)$ and at the third one by $\left(a, \frac{1}{n} ; b, \frac{1}{n} ; c, 1-\frac{2}{n}\right)$.

Then we come to the probabilities with which P1 chooses his actions from the various information sets belonging to him. From $I_{1}^{1}$ and $I_{1}^{2}$ the choices and the probabilities attached to these are $\left(A_{1}, 1-\frac{1}{n} ; c_{1}, \frac{1}{n}\right)$, and from $I_{1}^{3}$, as well as from all the singletons, they are $\left(A_{1}, \frac{1}{n} ; c_{1}, 1-\frac{1}{n}\right)$.

With respect to $\mathrm{P} 2$ choices and probabilities are given as follows. From $I_{2}^{1}$ and $I_{2}^{6}$ they are $\left(A_{2}, 1-\frac{1}{n} ; b_{2}, \frac{1}{n}\right)$ and from $I_{2}^{2}, I_{2}^{3}, I_{2}^{4}$ and $I_{2}^{5}$ they are $\left(A_{2}, \frac{1}{n} ; b_{2}, 1-\frac{1}{n}\right)$. With respect to the singletons belonging to $\mathrm{P} 2$ we have for all of them $\left(A_{2}, \frac{1}{n} ; b_{2}\right.$, $\left.1-\frac{1}{n}\right)$.

Beliefs are indicated by the probabilities attached to the nodes of the information sets. Below by the left (right) probability we mean the consistent with the above behavioral strategies belief that the player attaches to being at the left (right) corner node of an information set. We also give the limit of these beliefs as $n$ tends to $\infty$.

In $I_{1}^{1}$ the left probability is $\frac{1-\frac{2}{n}}{1-\frac{1}{n}}$ and the right probability is $\frac{\frac{1}{n}}{1-\frac{1}{n}}$. The limit is $(1,0)$.

In $I_{1}^{2}$ the left probability is $\frac{\frac{1}{n}}{1-\frac{1}{n}}$ and the right probability is $\frac{1-\frac{2}{n}}{1-\frac{1}{n}}$. The limit is $(0,1)$.

In $I_{1}^{3}$ the left probability is $\frac{1}{2}$ and the right probability is $\frac{1}{2}$. The limit is $\left(\frac{1}{2}, \frac{1}{2}\right)$.

In $I_{2}^{1}$ the left probability is $\frac{\left(1-\frac{1}{n}\right)\left(1-\frac{2}{n}\right)}{\left(1-\frac{2}{n}\right)\left(1-\frac{1}{n}\right)+\left(\frac{1}{n}\right)^{2}}$ and the right probability is $\frac{\left(\frac{1}{n}\right)^{2}}{\left(1-\frac{1}{n}\right)\left(1-\frac{2}{n}\right)+\left(\frac{1}{n}\right)^{2}}$. The limit is $(1,0)$.

In $I_{2}^{2}$ the left probability is $\frac{\left(1-\frac{2}{n}\right) \frac{1}{n}}{\left(1-\frac{2}{n}\right) \frac{1}{n}+\left(1-\frac{1}{n}\right) \frac{1}{n}}$ and the right probability is $\frac{\left(1-\frac{1}{n}\right) \frac{1}{n}}{\left(1-\frac{2}{n}\right) \frac{1}{n}+\left(1-\frac{1}{n}\right)\left(\frac{1}{n}\right)}$. The limit is $\left(\frac{1}{2}, \frac{1}{2}\right)$. 
In $I_{2}^{3}$ the left probability is $\frac{\left(1-\frac{1}{n}\right) \frac{1}{n}}{\left(1-\frac{1}{n}\right) \frac{1}{n}+\left(\frac{1}{n}\right)^{2}}$ and the right probability is $\frac{\left(\frac{1}{n}\right)^{2}}{\left(1-\frac{1}{n}\right) \frac{1}{n}+\left(\frac{1}{n}\right)^{2}}$. The limit is $(1,0)$.

In $I_{2}^{4}$ the left probability is $\frac{\left(\frac{1}{n}\right)^{2}}{\left(1-\frac{1}{n}\right) \frac{1}{n}+\left(\frac{1}{n}\right)^{2}}$ and the right probability is $\frac{\left(1-\frac{1}{n}\right) \frac{1}{n}}{\left(1-\frac{1}{n}\right) \frac{1}{n}+\left(\frac{1}{n}\right)^{2}}$. The limit is $(0,1)$.

In $I_{2}^{5}$ the left probability is $\frac{\left(\frac{1}{n}\right)^{2}}{\left(1-\frac{2}{n}\right) \frac{1}{n}+\left(\frac{1}{n}\right)^{2}}$ and the right probability is $\frac{\left(1-\frac{2}{n}\right) \frac{1}{n}}{\left(1-\frac{2}{n}\right) \frac{1}{n}+\left(\frac{1}{n}\right)^{2}}$. The limit is $(0,1)$.

In $I_{2}^{6}$ the left probability is $\frac{\left(1-\frac{1}{n}\right) \frac{1}{n}}{\left(1-\frac{1}{n}\right) \frac{1}{n}+\left(1-\frac{1}{n}\right)\left(1-\frac{2}{n}\right)}$ and the right probability is $\frac{\left(1-\frac{1}{n}\right)\left(1-\frac{2}{n}\right)}{\left(1-\frac{1}{n}\right) \frac{1}{n}+\left(1-\frac{1}{n}\right)\left(1-\frac{2}{n}\right)}$. The limit is $(0,1)$.

The belief attached to each singleton is that it has been reached with probability 1 .

The limits of the sequence of strategies and beliefs confirm a particular Bayesian equilibrium as a sequential one. In an analogous manner, sequential equilibria can also be obtained for the models analyzed in the previous sections.

\section{Concluding remarks}

As we have already emphasized in Glycopantis, Muir, and Yannelis (2001), we consider the area of incomplete and differential information and its modelling important for the development of economic theory. We believe that the introduction of game trees, which gives a dynamic dimension to the analysis, helps in the development of ideas.

The discussion in that paper is in the context of one-good examples without free disposal. The conclusion was that core notions which may not be CBIC, such as the weak fine core, cannot easily be supported as a PBE. On the other hand, in the presence of an agent with superior information, the private core which is CBIC can be supported as a PBE. The discussion provided a noncooperative interpretation or foundation of the private core while making, through the game tree, the individual decisions transparent. In this way a better understanding of how incentive compatible contracts are formed is obtained.

In the present paper we investigate, in a one-good, two-agent economy, with and without free disposal, the implementation of private core, of Radner equilibrium, of weak fine core and weak fine values allocations. We obtain, through the construction of a tree with reasonable rules, that free disposal private core allocations, to which also the Radner equilibrium belongs, are not implementable. A brief comparison 
of the idea of CBIC in the static presentation with the case when the analysis is in terms of game trees is made.

It is surprising that free disposal destroys incentive compatibility and creates problems for implementation. Implementation in this case can be achieved by invoking an exogenous third party which can be thought of as a court that penalizes lying agents. It is of course possible that rational agents, once they realize that they can be cheated, might decide not to trade rather than rely on a third party which has to prove that he has perfect knowledge and can execute the correct trades. Notice that the third, exogenous party, in this case the court, plays the role of the mechanism designer in the relevant implementation literature (see Hahn and Yannelis, 2001, and the references there).

Similarly, implementation of a private core allocation becomes possible through the introduction of an endogenous third party with zero endowments but with superior information. In this case the third party is part of the model, i.e. an agent whose superior information allows him to play the role of an intermediary. The analysis overlaps with the one in Glycopantis, Muir, and Yannelis (2001). On the other hand we show here that implementation can also be achieved through a sequential equilibrium. It should be noted that the endogenous third agent is rewarded for his superior information by receiving consumption in a particular state, in spite of the fact that he has zero initial endowments in each state. However, both Radner equilibria and REE would not recognize a special role to such an agent. These Walrasian type notions would award to him zero consumption in all states of nature.

In summary, the analysis here considers the relation between, cooperative and noncooperative, static equilibrium concepts and noncooperative, game theoretic dynamic processes in the form of game trees. We have examined the possible support and implementation as perfect Bayesian equilibria of the cooperative concepts of the private core and the weak fine core, and the noncooperative generalized, Walrasian type equilibrium notions of Radner equilibrium and REE. In effect what we are doing is to look directly into the Bayesian incentive compatibility of the corresponding allocations, as if they were contracts, and then consider their implementability.

\section{Appendix I: A note on PBE}

In this note we look briefly at equilibrium notions when sequential decisions are taken by the players, i.e. in the context of game trees. For strategies we shall employ the idea of a behavioral strategy for a player being an assignment to each of his information sets of a probability distribution over the options available from that set. For a game of perfect recall, Kuhn (1953) shows that analysis of the game in terms of behavioral strategies is equivalent to that in terms of, the more familiar, mixed strategies. In any case, behavioral strategies are more natural to employ with an extensive form game. Sometimes we shall refer to them simply as strategies.

Consider an extensive form game and a given profile of behavioral strategies

where $I$ is the set of players.

$$
s=\left\{s_{i}: i \in I\right\}
$$


When $s$ is used each node of the tree is reached with probability obtained by producting the option probabilities given by $s$ along the path leading to that node. In particular, there is a probability distribution over the set of terminal nodes so the expected payoff $E_{i}$ to each player Pi may be expressed in terms of option probabilities from each information set.

Consider any single information set $J$ owned by $P i$, with corresponding option probabilities $\left(1-\pi_{J}, \pi_{J}\right)$, where for simplicity of notation we assume binary choice. The dependence of $E_{i}$ on $\pi_{J}$ is determined only by the paths which pass through $J$. Taking any one of these paths, on the assumption that the game is of perfect recall, the term it contributes to $E_{i}$ will only involve $\pi_{J}$ once in the corresponding product of probabilities. Thus, on summing over all such paths, the dependence of $E_{i}$ on $\pi_{J}$ is seen to be linear, with coefficients depending on the remaining components of $s$.

This allows the formation of a reaction function expressing $\pi_{J}$ in terms of the remaining option probabilities, by optimizing $\pi_{J}$ while holding the other probabilities constant; hence the Nash equilibria are obtained, as usual, as simultaneous solutions of all these functional relations. We are here adopting an agent form for a player, where optimization with respect to each of his decisions is done independently from all the others. A solution is guaranteed by the usual proof of existence for Nash equilibria.

For example, consider the tree in Figure 4, denoting the option probabilities from $I_{1}, I_{2}$ by $(1-\alpha, \alpha),(1-\beta, \beta)$ respectively. The payoff functions are then (apart from the factor $\frac{1}{3}$ expressing the probability of Nature's choice, and leaving out terms not involving $\alpha$ which come from paths not passing through $I_{1}, I_{2}$

$$
\begin{aligned}
E_{1}= & 5(1-\alpha)(1-\beta)+5(1-\alpha) \beta \\
& +4 \alpha(1-\beta)+4 \alpha \beta+2.5(1-\alpha)+4 \alpha+\ldots \\
= & 7.5+0.5 \alpha+\ldots ; \\
E_{2}= & 5(1-\alpha)(1-\beta)+4(1-\alpha) \beta \\
& +5 \alpha(1-\beta)+4 \alpha \beta+2.5(1-\beta)+4 \beta+\ldots \\
= & 7.5+0.5 \beta+\ldots
\end{aligned}
$$

Since the coefficient of $\alpha$ in $E_{1}$ is positive, the optimal choice of $\alpha$, i.e. the reaction function of Agent 1 is 1 . Similarly for $\beta$ in $E_{2}$ we obtain the value 1, and this is the reaction function of Agent 2.

Note that in any such calculation, only the coefficient of each $\pi_{J}$ is important for the optimization - the rest of $E_{i}$ is irrelevant. We may similarly treat the 21 option probabilities in Figure 7, obtaining 21 conditions which they must satisfy. These are quite complex and there are, probably, many solutions but it may be checked that the one given satisfies all conditions.

When an equilibrium profile is used, it is possible that some nodes are visited with zero probability. This means that the restriction of the strategy profile to subsequent nodes has no effect on the expected payoffs, so may be chosen arbitrarily. To eliminate this redundancy in the set of Nash equilibria, a refinement of the equilibrium concept to that of perfect equilibrium, was introduced for games of perfect information - that is, games in which each information set is a singleton. 
This requires an equilibrium strategy also to be a Nash equilibrium for all subgames of the given game. In other words, the strategy profile should be a Nash equilibrium for the game which might be started from any node of the given tree, not just the nodes actually visited in the full game.

Any attempt to extend this notion to general games encounters the problem that sub-trees might start from nodes which are not in singleton information sets. In such a case, the player who must move first cannot know for certain at which node he is located within that set. He can only proceed if he adopts beliefs about where he might be, in the form of a probability distribution over the nodes of the information set. Moreover, these beliefs must be common knowledge, for the other players to be able to respond appropriately, so the desired extension of the equilibrium concept must take into account both strategies and beliefs of the players. The game will be played from any information set as if the belief probabilities had been realised by an act of nature.

We need, therefore, to consider pairs $(s, \mu)$, consisting of a behavioral strategy profile $s$ and a belief profile

$$
\mu=\left\{\mu_{J}: J \in \mathcal{J}\right\}
$$

Here, $\mathcal{J}$ denotes the set of information sets and $\mu_{J}$ is a probability distribution over the nodes of information set $J$, expressing the beliefs of the player who might be required to play from that set. Given the belief profile, we then require that the strategy profile give a perfect equilibrium, in the sense of being optimal for each player starting from every information set. But we need also to consider the source of the beliefs.

Given any behavioral strategy profile $s$ denote the probability of reaching any node $a$, using $s$, by $\nu(a)$. Consider first an information set, $J$, not all of whose nodes are visited with zero probability when using $s$. We may calculate the conditional probability of being at a node $a \in J$ given that it is in $J$ by

$$
\nu(a \mid J)=\frac{\nu(\{a\} \cap J)}{\nu(J)}=\frac{\nu(a)}{\nu(J)}
$$

since $a \in J \Rightarrow\{a\} \cap J=\{a\}$. Thus the belief probabilities $\mu_{J}(a)=\nu(a \mid J)$ for $J$ are just the relative probabilities of reaching the nodes of $J$.

For example, returning to Figure 4 and employing the only Nash solution $\alpha=$ $\beta=1$ noted above, the probabilities of reaching the nodes of $I_{2}$ are $0, \frac{1}{3}, \frac{1}{3}$ which relativises, given the condition that we reach $I$, to $0, \frac{1}{2}, \frac{1}{2}$ as stated.

Thus for a $P B E$, the behavioral strategy-belief profile pair $(s, \mu)$ should satisfy two conditions:

(i) For the given belief profile $\mu$, the strategy profile $s$ should be a perfect equilibrium, as defined above;

(ii) For the given strategy profile $s$, the belief profile $\mu$ should be calculated at each information set for which $\nu(I) \neq 0$ by the formula above.

Justifications of the concept of perfect equilibrium in games of perfect information will argue that the players need to have good strategies to employ, even were something to go wrong with the intended play so that the game accidentally 
enters sub-trees which ought not to be accessed. One way to argue this is through the notion of a trembling hand which makes errors, so possibly choosing the wrong move. Employing this same idea in the context of perfect Bayesian equilibria, we can allow small perturbations in the strategies, such that all information sets are visited with non-zero probability. Then the relation determining beliefs from strategies is well posed and we may consider only beliefs which arise as limiting cases of such perturbations. This more restrictive definition of equilibrium is called a sequential equilibrium.

\section{References}

Allen, B.: Generic existence of completely revealing equilibria with uncertainty, when prices convey information. Econometrica 49, 1173-1199 (1986)

Allen, B., Yannelis, N. C.: Differential information economies. Economic Theory 18, 263-273 (2001)

Aumann, R. J.: Correlated equilibria as an expression of Bayesian rationality. Econometrica 55, 1-18 (1987)

Emmons, D., Scafuri, A. J.: Value allocations - An exposition. In: Aliprantis, C. D., Burkinshaw, O., Rothman, N. J. (eds.) Advances in economic theory. Lecture notes in economics and mathematical systems, pp. 55-78. Berlin Heidelberg New York: Springer 1985

Einy, E., Moreno, D., Shitovitz, B.: Rational expectations equilibria and the ex post core of an economy with asymmetric information. Journal of Mathematical Economics 34, 527-535 (2000)

Einy, E., Moreno, D., Shitovitz, B.: Competitive and core allocations in large economies with differential information. Economic Theory 34, 321-332 (2001)

Glycopantis, D., Muir, A., Yannelis, N. C.: An extensive form interpretation of the private core. Economic Theory 18, 293-319 (2001)

Glycopantis, D., Muir, A., Yannelis, N.C.: On the extensive form implementation of REE. Mimeo (2002)

Hahn, G., Yannelis, N. C.: Efficiency and incentive compatibility in differential information economies. Economic Theory 10, 383-411 (1997)

Hahn, G., Yannelis, N.C.: Coalitional Bayesian Nash implementation in differential information economies. Economic Theory 18, 485-509 (2001)

Koutsougeras, L., Yannelis, N. C.: Incentive compatibility and information superiority of the core of an economy with differential information. Economic Theory 3, 195-216 (1993)

Krasa, S., Yannelis, N.C.: The value allocation of an economy with differential information. Econometrica 62, 881-900 (1994)

Kreps, M. D., Wilson, R.: Sequential equilibrium. Econometrica 50, 889-904 (1982)

Kuhn, H. W.: Extensive games and the problem of information. In: Kuhn, H. W., Tucker, A. W. (eds.) Contributions to the theory of games, Vol. II. Annals of mathematical studies, Vol. 28, pp. 193-216. Princeton, NJ: Princeton University Press 1953

Kurz, M.: On rational belief equilibria. Economic Theory 4, 859-876 (1994)

Radner, R.: Competitive equilibrium under uncertainty. Econometrica 36, 31-58 (1968)

Scafuri, A. J., Yannelis, N. C.: Non-symmetric cardinal value allocations. Econometrica 52, 1365-1368 (1984)

Shapley, L. S.: A value for n-person games. In: Kuhn, H. W., Tucker, A. W. (eds.) Contributions to the theory of games, Vol. II. Annals of mathematical studies, Vol. 28, pp. 307-317. Princeton, NJ: Princeton University Press 1953

Tirole, J.: The theory of industrial organization. Cambridge, MA London: The MIT Press 1988

Wilson, R.: Information, efficiency, and the core of an economy. Econometrica 46, 807-816 (1978)

Yannelis, N. C.: The core of an economy with differential information. Economic Theory 1, 183-198 (1991) 